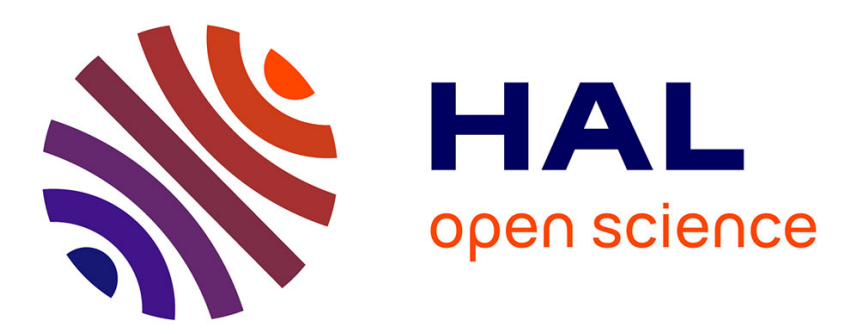

\title{
Three-dimensional scour simulations with a two-phase flow model
}

Tim Nagel, Julien Chauchat, Cyrille Bonamy, Xiaofeng Liu, Zhen Cheng, Tian-Jian Hsu

\section{- To cite this version:}

Tim Nagel, Julien Chauchat, Cyrille Bonamy, Xiaofeng Liu, Zhen Cheng, et al.. Three-dimensional scour simulations with a two-phase flow model. Advances in Water Resources, 2020, 138, pp.103544. 10.1016/j.advwatres.2020.103544 . hal-02500524

\section{HAL Id: hal-02500524 \\ https://hal.science/hal-02500524}

Submitted on 12 Mar 2020

HAL is a multi-disciplinary open access archive for the deposit and dissemination of scientific research documents, whether they are published or not. The documents may come from teaching and research institutions in France or abroad, or from public or private research centers.
L'archive ouverte pluridisciplinaire HAL, est destinée au dépôt et à la diffusion de documents scientifiques de niveau recherche, publiés ou non, émanant des établissements d'enseignement et de recherche français ou étrangers, des laboratoires publics ou privés. 


\title{
Three-dimensional scour simulations with a two-phase flow model
}

\author{
Tim Nagel ${ }^{\mathrm{a}, \mathrm{b}}$, Julien Chauchat ${ }^{\mathrm{a}, \mathrm{b}, *}$, Cyrille Bonamy ${ }^{\mathrm{a}, \mathrm{b}}$, Xiaofeng Liư ${ }^{\mathrm{c}}$, Zhen Cheng ${ }^{\mathrm{d}, \mathrm{e}}$, Tian-Jian \\ $\mathrm{Hsu}^{\mathrm{d}}$ \\ ${ }^{a}$ Univ. Grenoble Alpes, LEGI, F-38000 Grenoble, France. \\ ${ }^{b}$ CNRS, LEGI, F-38000 Grenoble, France. \\ ${ }^{c}$ Dept. of Civil and Environmental Engineering, Institute of CyberScience, Pennsylvania State Univ., State College, PA \\ 16802, USA. \\ ${ }^{d}$ Center for Applied Coastal Research, University of Delaware, Newark, DE 19716, USA. \\ ${ }^{e}$ Now at Applied Ocean Physics $\mathcal{E}$ Engineering, Woods Hole Oceanographic Institution, Woods Hole, MA 02543, USA.
}

\begin{abstract}
In this contribution, a three-dimensional sediment scour model based on the two-phase flow Eulerian-Eulerian solver, sedFoam, is developed within the framework of the open source platform OpenFOAM. The adoption of the Eulerian approach for both fluid and sediment makes the model suitable for simulating scour process around structures with arbitrary geometry. The model is first validated with unidirectional sediment transport configurations without structures, to ensure that it can capture both flow and sediment transport processes. The validation cases show that the model can accurately simulate the sediment transport rate and bedload layer thickness as a function of the Shields number over a wide range of flow condition and sediment properties. Then, the model is applied to simulate the live-bed scour process around a vertical pile due to unidirectional current. It is a first attempt to use a two-phase model for simulating such case and its success serves as a proof-of-concept for future development. Simulated results of flow, sediment transport, and scour processes are compared with experiments and good agreement is observed. A new methodology to determine the bed shear stress in complex flow configurations is proposed. The mixture shear stress computed at the elevation of the iso-concentration $\phi_{0}=0.08$ (corresponding to top of the bedload layer) is used to define the local Shields number. Within the scour hole, a competition between fluid bed shear stress driven and gravity driven sediment transport occurs at bed angles up to $\beta \approx 23^{\circ}$. This is lower than the repose angle $\left(\beta_{r}=32^{\circ}\right)$. The relationship between the sediment flux and the bed slope is linear below $\beta \approx 23^{\circ}$. Above this angle the sediment flux increases nonlinearly with the bed slope while the Shields number tends to 0 . This shows that, in the part of the scour hole close to the vertical cylinder, the sediment flux only result from the action of gravity: avalanching is the dominant transport mechanism and conventional power law become ineffective even with slope correction. In the lee-side of the cylinder suspended load dominant and the power law, assuming a local equilibrium between bottom shear stress and transport flux, is no longer valid. Further discussion is made on the computational cost of the proposed model and future research directions.
\end{abstract}

Keywords: Two-phase flow, scour, numerical modeling

\footnotetext{
*Corresponding author at LEGI UMR 5519, BP 53, 38041 Grenoble Cedex 9, France.

Email address: julien. chauchat@univ-grenoble-alpes.fr (Julien Chauchat) 


\section{Introduction}

Numerical simulation of scour is a very difficult task due to several scientific and technical obstacles. First, sediment transport is an extremely complex phenomena involving many physical processes, e.g. particle-particle, fluid-particle and turbulence-particle interactions, for which existing models exhibit limited predictive capability. An factor two error in sediment fluxes is often considered reasonable for steady and uniform flow conditions [Gomez and Church, 1989]. When the fluid flow is three-dimensional, unsteady and non-uniform, such as in the scour configuration, existing models for sediment fluxes are questionable because they assume a local equilibrium between the bottom shear stress ans the sediment transport flux. Second, when scour occurs, the fluid flow is almost always turbulent and the flow dynamics is complex with turbulent structures such as the horse-shoe vortex (HSV) system upstream or vortex shedding downstream of an obstacle. Even though turbulence modeling has made significant progress over the last decades, this remains one of the major unsolved problems in fluid dynamics. From a technical point of view, scour is a rather large scale problem for high-resolution numerical simulation. The domain size has to be large enough to capture the vortex shedding correctly and the erosion process takes place at long time scale which makes the simulations very challenging. The third technical difficulty is that the scour process involves a dynamically changing interface, i.e., the bed, and its interaction with the stationary structure. For structures with complex geometry, the dynamic intersection of the bed surface and the structure is extremely difficult to capture. There are several techniques to deal with the problem, for example the Arbitrary-Lagrangian-Eulerian approach (ALE, also termed the moving mesh method), the Eulerian approach, and the immersed boundary method. The method introduced in this paper belongs to the Eulerian approach where water and sediment are treated with a two-phase flow model. The development and application of scour models have also been hindered by the excessive computational resources required for such simulations.

In the literature, most of the scour models use what is denoted here as the "classical" approach where they couple the hydrodynamics (solving the Navier-Stokes equations) and the bed morphodynamics (solving the Exner's equation [Exner, 1920, 1925]). Such coupling approach can be best demonstrated by the work of Roulund et al. [2005]. In their model, the bottom stress was first calculated based on the simulated flow field. Then, the bottom shear was used to calculate the sediment transport rate, which was consequently used in the Exner equation for updating the new bed position. With this approach, Roulund et al. [2005] show that the agreement between the numerical predictions and the experimental observations is good in both transient and equilibrium states. The numerical simulations presented in Roulund et al. [2005] are often considered as a reference case in many posterior studies [Stahlmann et al., 2013; Baykal et al., 2015]. These cases are also considered in this work.

The "classical" approach for scour modeling used in Roulund et al. [2005] or Baykal et al. [2015] is based on empirical sediment transport formulas developed for steady and uniform flow conditions [Meyer-Peter and Müller, 1948; Engelund and Fredsøe, 1976]. In the presence of an obstacle, such as a bridge pier, the flow is highly unsteady, non-uniform and the bed slope can reach values near the angle of repose. As a result, most formulas are used beyond their validity range when applied to three-dimensional scour around structures. Furthermore, to avoid the slope angle in scour hole to be greater than the angle of repose, ad-hoc avalanche models are always applied, which essentially redistribute sediment mass by a smoothing scheme. Most of these avalanche models have very little physical bases and more studies are warranted.

In the simple configuration of a single pile, the performance of classical models are satis- 
factory [Roulund et al., 2005; Stahlmann et al., 2013; Baykal et al., 2015]. However, to design civil engineering structures, the conventional sediment transport models are not comprehensive enough. Therefore, engineers are still mainly relying on small-scale physical models.

To overcome the limitations of classical models for scour, two-phase flow models have been developed over the last decade [Amoudry et al., 2008; Cheng et al., 2017; Mathieu et al., 2019]. In this new framework, the sediment phase can be either modeled as discrete particles (termed the Eulerian-Lagrangian approach), or as a continuous phase (termed the Eulerian-Eulerian approach). In both approaches, the fluid phase is always seen as a continuum. In the Lagrangian approach, a dynamical equation is solved for each individual particle and its interactions with neighboring particles [e.g., Escauriaza and Sotiropoulos, 2011]. The computational resources required by this approach are too expensive, at present, for engineering applications. Nevertheless, some aspects of scour can be studied using the Lagrangian approach. For example, Link et al. [2012] used the model of Escauriaza and Sotiropoulos [2011] with 10,000 particles to investigate the sediment transport capacity by the horseshoe vortex in a non-erodible scour hole. In their approach, the particles were considered as point particles and the fluid flow around them was not explicitly solved.

In the Eulerian approach for the sediment phase, sediment is treated as a complex fluid with a peculiar rheology interacting with water through buoyancy and drag forces. Such model has been applied with some success to various configurations ranging from sheet-flow [Jenkins and Hanes, 1998; Hsu et al., 2004; Cheng et al., 2017; Chauchat, 2018] to 2D scour configurations downstream of an apron [Amoudry and Liu, 2009; Cheng et al., 2017; Chauchat et al., 2017], and scour under a pipeline [Lee et al., 2016; Mathieu et al., 2019].

In this paper, we present the first three-dimensional Eulerian-Eulerian two-phase flow simulation of the 3D scour around a vertical cylinder. The first objective of the present work is to provide the proof of concept that the two-phase flow approach can be used for modeling the scour phenomenon. The main advantage of such approach is that it can intrisically deal with complex structures and does not rely on any empirical sediment transport formula or avalanching model. The second objective is to evaluate the assumption of a local correlation between the sediment transport flux and the fluid bed shear stress in order to evaluate the main assumptions routinely used by conventional scour models.

The paper is organized as follows. In Section 2, the mathematical formulation of the EulerianEulerian two-phase flow model is presented. In Section 3, the model is calibrated first with a steady, uniform and unidirectional sediment transport configuration with medium sand. In Section 7, the hydrodynamic validation of the flow around a vertical cylinder mounted on a flat non-erodible bed is undertaken. In Section 4, the results of the three-dimensional scour simulation are presented with a validation against experimental data. An in-depth discussion on the sediment transport around the pile is provided in Section 6. At the end, a summary of findings is given and some future directions are discussed.

\section{Mathematical Model}

The mathematical formulation of the Eulerian-Eulerian two-phase flow model has been detailed in Chauchat et al. [2017]. The model is obtained by averaging the local and instantaneous mass and momentum conservation equations over fluid and dispersed particles [e.g., Hsu et al., 2004]. The resulting system of governing equations can be considered as the fundamental equations for two-phase flow system similar to the Navier-Stokes equations for single-phase clear fluid system. When applying these equations to turbulent flow, additional turbulence averaging, 
or filtering, has to be used. In the present model, the turbulence-averaged Eulerian two-phase flow equations described in Chauchat et al. [2017] are used and a new closure is developed.

\subsection{Two-phase flow model equations for incompressible fluid}

The mass conservation equations for the particle phase and fluid phase are written as:

$$
\begin{gathered}
\frac{\partial \phi}{\partial t}+\frac{\partial \phi u_{i}^{s}}{\partial x_{i}}=0, \\
\frac{\partial(1-\phi)}{\partial t}+\frac{\partial(1-\phi) u_{i}^{f}}{\partial x_{i}}=0,
\end{gathered}
$$

where $\phi$, and $1-\phi$ are the particle and fluid volume fractions, $u_{i}^{s}, u_{i}^{f}$ are the sediment and fluid phase velocities, and $i=1,2,3$ represents streamwise, spanwise and vertical component, respectively. Following Chauchat et al. [2017], the momentum equations for fluid and particle phases can be written as:

$$
\begin{aligned}
\frac{\partial \rho^{s} \phi u_{i}^{s}}{\partial t}+\frac{\partial \rho^{s} \phi u_{i}^{s} u_{j}^{s}}{\partial x_{j}}=-\phi \frac{\partial p}{\partial x_{i}}+\phi f_{i}-\frac{\partial \tilde{p}^{s}}{\partial x_{i}}+\frac{\partial \tau_{i j}^{s}}{\partial x_{j}}+\phi \rho^{s} g_{i}+\phi(1-\phi) K\left(u_{i}^{f}-u_{i}^{s}\right) & -\frac{1}{S_{c}}(1-\phi) K v_{t}^{f} \frac{\partial \phi}{\partial x_{i}} \\
\frac{\partial \rho^{f}(1-\phi) u_{i}^{f}}{\partial t}+\frac{\partial \rho^{f}(1-\phi) u_{i}^{f} u_{j}^{f}}{\partial x_{j}}= & -(1-\phi) \frac{\partial p}{\partial x_{i}}+(1-\phi) f_{i}+\frac{\partial \tau_{i j}^{f}}{\partial x_{j}} \\
& +(1-\phi) \rho^{f} g_{i}-\phi(1-\phi) K\left(u_{i}^{f}-u_{i}^{s}\right) \\
& +\frac{1}{S_{c}}(1-\phi) K v_{t}^{f} \frac{\partial \phi}{\partial x_{i}}
\end{aligned}
$$

where $\rho^{s}, \rho^{f}$ are the particle and the fluid density, respectively, $g_{i}$ is the gravitational acceleration and $p$ is the fluid pressure. $f_{i}$ is the external force that drives the flow. The fluid stress $\tau_{i j}^{f}$ includes fluid grain-scale (viscous) stress and fluid Reynolds stresses, $\tilde{p}^{s}, \tau_{i j}^{s}$ are particle normal stress and shear stress. The last two terms on the right-hand-side (RHS) of Eqn. (3) and and Eqn. (4) are momentum coupling between the fluid phase and particle phase through drag force, where $K$ is the drag parameter. The second to the last term represents averaged drag force due to mean relative velocity between fluid and particle phases, while the last term represents the fluid turbulent suspension term, also called drift velocity [Deutsch and Simonin, 1991]. Finally, $v_{t}^{f}$ is the turbulent viscosity that has to be calculated using a turbulence closure and $S_{c}$ is the Schmidt number.

The drag parameter $K$, is modeled following Schiller and Naumann [1933]:

$$
K=0.75 C_{d} \frac{\rho^{f}}{d}\left\|\boldsymbol{u}^{f}-\boldsymbol{u}^{s}\right\|(1-\phi)^{-h_{E x p}}
$$

where $d$ is the particle diameter and $\boldsymbol{u}^{k}$ the velocity vector of the phase $\mathrm{k}$. The hindrance function $(1-\phi)^{-h_{E x p}}$ represents the drag increase when the particle volume fraction increases. $h_{E x p}=2.65$ is the hindrance exponent that depends on the particulate Reynolds number. Here, following 
Chauchat et al. [2017] its value is constant.

The drag coefficient $C_{d}$ is calculated as:

$$
C_{d}=\left\{\begin{array}{r}
\frac{24}{R e_{p}}\left(1+0.15 R e_{p}^{0.687}\right), R e_{p} \leq 1000 \\
0.44, R e_{p}>1000
\end{array},\right.
$$

in which, the particulate Reynolds number $R e_{p}$ is defined as: $R e_{p}=(1-\phi)\left\|\boldsymbol{u}^{f}-\boldsymbol{u}^{s}\right\| d / v^{f}$, where $v^{f}$ represents the fluid kinematic viscosity.

\subsection{Fluid phase shear stress}

Due to the fact that the present model equations are obtained by averaging over turbulence, the fluid stresses consist in a fluid-phase component $R_{i j}^{f}$ (i.e., fluid-phase Reynolds stress) and a grain-scale stress $r_{i j}^{f}$, including the viscous stress and an additional effect due to fluid-particle interaction at the grain scale. The total fluid stress is written as:

$$
\begin{gathered}
\tau_{i j}^{f}=R_{i j}^{f}+r_{i j}^{f}, \\
R_{i j}^{f}=\rho^{f}(1-\phi)\left[2 v_{t}^{f} S_{i j}^{f}-\frac{2}{3} k \delta_{i j}\right], \\
r_{i j}^{f}=2 \rho^{f}(1-\phi) v^{m i x} S_{i j}^{f},
\end{gathered}
$$

where $v_{t}^{f}$ is the eddy viscosity, $k$ is the turbulent kinetic energy and $v^{m i x}$ is the mixture viscosity, defined as a function of the solid volume fraction as proposed by Boyer et al. [2011] (see Chauchat et al. [2017] for more details). $S_{i j}^{f}$ is the deviatoric part of the fluid phase strain rate tensor and is defined as:

$$
S_{i j}^{f}=\frac{1}{2}\left(\frac{\partial u_{i}^{f}}{\partial x_{j}}+\frac{\partial u_{j}^{f}}{\partial x_{i}}\right)-\frac{1}{3} \frac{\partial u_{k}^{f}}{\partial x_{k}} \delta_{i j} .
$$

\subsection{Turbulence modeling}

The two-phase flow turbulence averaged formulation requires a closure for the eddy viscosity. Several turbulence models are available in sedFoam, including a two-phase version of the $\mathrm{k}-\varepsilon$ turbulence model [Cheng et al., 2017] and a two phase version of the $\mathrm{k}-\omega$ turbulence model [Chauchat et al., 2017]. However, it is known that these turbulence models are not accurate in the case of boundary-layer flows with a strong adverse pressure gradient (such as the flow around a vertical cylinder) and the use of the k- $\omega$ SST developed by Menter [1993] is recommended in Roulund et al. [2005]. It was proven difficult if not impossible to adapt the k- $\omega$ SST model to two-phase flows. Indeed, in this turbulence model blending functions are introduced for some of the model coefficients. These blending functions depend on the distance to solid boundaries that is not clearly defined in the presence of a mobile sediment bed.

Recently, a reformulated version of the standard k- $\omega$ has been developed (see Wilcox [2006, 2008]) and successfully applied to the scour around a cylindrical pile case by Baykal et al. [2015]. This "revisited" model formulation incorporates a cross-diffusion term and a built-in stress-limiter modification to behave as the SST model. Its adaptation to a two-phase flow version can be done similarly to what is presented in Chauchat et al. [2017] for the standard k- $\omega$ turbulence model. This new model will be denoted as the two-phase k- $\omega 2006$ in the present 
paper.

First, the Turbulent Kinetic Energy (TKE) $k$ is computed from the solution of Eqn. (11), appropriate for sand particles in water [Hsu et al., 2004; Yu et al., 2010]:

$$
\begin{aligned}
\frac{\partial k}{\partial t}+u_{j}^{f} \frac{\partial k}{\partial x_{j}}= & R_{i j}^{f} \frac{\partial u_{i}^{f}}{\partial x_{j}}-C_{\mu} k \omega+\frac{\partial}{\partial x_{j}}\left[\left(v^{f}+\sigma_{k} v_{t}^{f}\right) \frac{\partial k}{\partial x_{j}}\right] \\
& -\frac{2 K\left(1-t_{m f}\right) \phi k}{\rho^{f}}-\frac{1}{S_{c}(1-\phi)} v_{t}^{f} \frac{\partial \phi}{\partial x_{j}}\left(\frac{\rho^{s}}{\rho^{f}}-1\right) g_{j}
\end{aligned}
$$

The above $k$-equation is similar to the clear fluid $\mathrm{k}-\omega 2006$ closure, the first three terms on the RHS describe respectively production, dissipation and diffusion of TKE. The last two terms on the RHS of Eqn. (11) describe the modification of the classical $k$-transport equation induced by the presence of particules. The fifth term on RHS accounts for the sediment damping effect on the carrier flow turbulence through density stratification. It can be seen as the buoyancy production/dissipation due to sediment-induced density stratification [Kranenburg et al., 2014].

The fourth term on RHS is a damping term modeling the drag-effect of sediment particles on the carrier flow turbulence. Indeed, if their inertia is important enough, particles cannot completely follow the turbulent fluid velocity fluctuations. In this drag-induced damping term, the parameter $t_{m f}$ is introduced to characterize the degree of correlation between particles and fluid velocity fluctuations. Following Kranenburg et al. [2014], its value varies between 0 and $1 . t_{m f}=1$ denotes particles following instantaneously the turbulent velocity fluctuations. In that case, the turbulence damping of the carrier fluid vanishes. Conversely, when $t_{m f}=0$ the particles velocity fluctuations are uncorrelated to the fluid turbulence and the turbulence damping term is at maximum. This situation corresponds to high Stokes numbers, i.e., the particle inertia is much higher than the fluid. Danon et al. [1977] and Chen and Wood [1985] proposed an exponential function for $t_{m f}$, which is also used in Cheng et al. [2017]:

$$
t_{m f}=e^{-B \cdot S t},
$$

where $B$ is an empirical coefficient. The degree of correlation between particles and fluid velocity fluctuations can be quantified by the Stokes number $S_{t}$ [Benavides and van Wachem, 2008]:

$$
S_{t}=\frac{t_{p}}{t_{l}}
$$

where $t_{p}=\rho^{s} /((1-\phi) K)$ is the particle response time, $t_{l}=k /(6 \varepsilon)$ is the characteristic time scale of energetic eddies.

In the present paper, the $\mathrm{k}-\omega 2006$ and the $\mathrm{k}-\varepsilon$ are used. Between both models, the transport equation for the turbulent energy dissipation rate together as the expression of the turbulent viscosity differ. 


\subsection{1. $k-\omega 2006$ model}

The fluid specific rate of turbulent energy dissipation $(\omega)$ equation reads:

$$
\begin{aligned}
\frac{\partial \omega}{\partial t}+u_{j}^{f} \frac{\partial \omega}{\partial x_{j}}= & \frac{C_{1 \omega}}{v_{t}^{f}} R_{i j}^{f} \frac{\partial u_{i}^{f}}{\partial x_{j}}-C_{2 \omega} \omega^{2}+\frac{\partial}{\partial x_{j}}\left[\left(v^{f}+\sigma_{\omega} v_{t}^{f}\right) \frac{\partial \omega}{\partial x_{j}}\right]+C D_{k \omega} \\
& -C_{3 \omega} \frac{2 K(1-t m f) \phi \omega}{\rho^{f}}-C_{4 \omega} \frac{1}{S_{c}} \frac{\omega}{k(1-\phi)} v_{t}^{f} \frac{\partial \phi}{\partial x_{j}}\left(\frac{\rho^{s}}{\rho^{f}}-1\right) g_{j} .
\end{aligned}
$$

The different coefficient values can be found in Table 1. The coefficients associated with the present two-equations closure are adopted from their clear fluid counterpart [Wilcox, 2008]. The last two terms on the RHS of eq. (14) account for the sediment damping effect on the fluid carrier flow turbulence through drag and density stratification, respectively. According to the numerical experiments described in Chauchat et al. [2017] for the two-phase k- $\omega$ turbulence model, the coefficient $C_{3 \omega}$ is chosen to be 0.35 . The coefficient associated with the buoyancy term $C_{4 \omega}=0$ is used in stably stratified condition, while it is set to 1 for unstably stratified condition. B is left as the only free model calibration parameter. From our experience, B can be tuned in the range of $[0.1-2]$. For instance, increasing B from 0.25 to 1.25 leads to an increase of the associated sediment flux of approximatively $20 \%$ on a simple sheet-flow case [Nagel, 2018]. All the simulations presented in this paper have been undertaken with the default value $\mathrm{B}=0.25$. The fourth term on the RHS of eq. (14), denoted as $C D_{k \omega}$ is a cross-diffusion term:

$$
C D_{k \omega}=A \frac{\sigma_{d}}{\omega} \frac{\partial k}{\partial x_{j}} \frac{\partial \omega}{\partial x_{j}},
$$

where the A coefficient is a smoothing term and $\sigma_{d}$ a coefficient that reads:

$$
\sigma_{d}=\mathcal{H}\left(\frac{\partial k}{\partial x_{j}} \frac{\partial \omega}{\partial x_{j}}\right) \sigma_{d 0},
$$

where $\sigma_{d 0}=1 / 8$ [Wilcox, 2008]. $\mathcal{H}($.$) is the Heaviside step function which has a value of unity$ if the argument is zero or positive and a value of zero if the argument is negative. The role of this cross-diffusion term is to increase the dissipation of TKE in the free-shear flow by enhancing the production of specific dissipation $\omega$ in that region. This will reduce the free-shear flow spreading rates sensitivity to the free-stream boundary conditions [Wilcox, 2008]. However it is important to suppress the cross-diffusion term near a solid boundary [Wilcox, 2006]. When approaching the wall $k$ and $\omega$ are respectively decreasing and increasing in the viscous sublayer. The argument of the Heaviside step function becomes negative and the cross-diffusion term is suppressed.

The A coefficient is a smoother imposing a gradual transition between the regions where the cross-diffusion term is activated or not. A is only present in the two-phase flow version of the model and allows to avoid instabilities:

$$
A=\frac{1}{2}(1+\tanh (-40(\phi-0.1))) \text {. }
$$

The coefficient involved in the dissipation of dissipation term (second term on the RHS of eq. (14)) follows the generalization of the Pope correction [Pope, 1988] given by Wilcox [2008]:

$$
\begin{aligned}
C_{2 \omega}= & C_{2 \omega 0} f_{C_{2 \omega}}, \\
& 7
\end{aligned}
$$


Table 1: Two phase $k-\omega 2006$ model coefficients.

\begin{tabular}{ccccccccc}
\hline$C_{\mu}$ & $C_{1 \omega}$ & $C_{2 \omega 0}$ & $C_{3 \omega}$ & $C_{4 \omega}$ & $\sigma_{k}$ & $\sigma_{\omega}$ & $\sigma_{d 0}$ & $S_{c}$ \\
\hline 0.09 & $5 / 9$ & 0.0708 & 0.35 & 1 & $3 / 5$ & 0.5 & $1 / 8$ & {$[1 / 3-1]$} \\
\hline
\end{tabular}

where

$$
C_{2 \omega 0}=0.0708, \quad f_{C_{2 \omega}}=\frac{1+85 \chi_{\omega}}{1+100 \chi_{\omega}},
$$

and

$$
\chi_{\omega}=\left|\frac{\Omega_{i j} \Omega_{j k} S_{k i}^{f}}{\left(C_{\mu} \omega\right)^{3}}\right|, \quad \Omega_{i j}=\frac{1}{2}\left(\frac{\partial u_{i}^{f}}{\partial x_{j}}-\frac{\partial u_{j}^{f}}{\partial x_{i}}\right) .
$$

The idea of Pope [1988] is to introduce the $\chi_{\omega}$ parameter to describe the vortex stretching. The latter is considered as the main mechanism for energy transfer from large to small eddies. Note that the Pope correction must be turned off for $1 \mathrm{D}$ or $2 \mathrm{D}$ configurations.

Finally, the turbulent eddy viscosity $v_{t}^{f}$ involved in $k$-equation and $\omega$-equation is calculated incorporating a stress-limiting term:

$$
v_{t}^{f}=\frac{k}{\tilde{\omega}}=\frac{k}{\max \left(\omega, C_{l i m} \sqrt{\frac{2 S_{i j}^{f} S_{i j}^{f}}{C_{\mu}}}\right)},
$$

where $C_{\text {lim }}=7 / 8$ [Wilcox, 2008].

\subsection{2. $k-\varepsilon$ model}

For the $k-\varepsilon$ model, the turbulent viscosity $v_{t}^{f}$ is calculated as:

$$
v_{t}^{f}=C_{\mu} \frac{k^{2}}{\varepsilon} .
$$

The dissipation rate $\varepsilon$ can be expressed in term of the specific dissipation rate $\omega$ as: $\varepsilon=C_{\mu} k \omega$. Therefore, for the $k-\varepsilon$ turbulence model, the TKE equation is similar to Eqn. (11) with the second term on the RHS replaced by $\varepsilon$.

The transport equation for the dissipation rate $\varepsilon$ reads:

$$
\begin{aligned}
\frac{\partial \varepsilon}{\partial t}+u_{j}^{f} \frac{\partial \varepsilon}{\partial x_{j}}= & C_{1 \varepsilon} \frac{\varepsilon}{k} \frac{R_{i j}^{f}}{\rho^{f}} \frac{\partial u_{i}^{f}}{\partial x_{j}}+\frac{\partial}{\partial x_{j}}\left[\left(v^{f}+\sigma_{\varepsilon} v_{t}^{f}\right) \frac{\partial \varepsilon}{\partial x_{j}}\right]-C_{2 \varepsilon} \frac{\varepsilon^{2}}{k} \\
& -C_{3 \varepsilon} \frac{\varepsilon}{k} \frac{2 K(1-t m f) \phi k}{\rho^{f}}-C_{4 \varepsilon} \frac{1}{S_{c}} \frac{\varepsilon}{k(1-\phi)} v_{t}^{f} \frac{\partial \phi}{\partial x_{j}}\left(\frac{\rho^{s}}{\rho^{f}}-1\right) g_{j} .
\end{aligned}
$$

The values of the empirical coefficients $\sigma_{\varepsilon}, C_{1 \varepsilon}, C_{2 \varepsilon}, C_{3 \varepsilon}, C_{4 \varepsilon}, C_{\mu}$ are listed in table 2 . The detailled description of the two-phase k- $\varepsilon$ turbulence model can be found in Hsu et al. [2004]; Cheng et al. [2017]; Chauchat et al. [2017]. 
Table 2: Two phase $k-\varepsilon$ turbulence model coefficients

\begin{tabular}{cccccc}
\hline$\sigma_{\varepsilon}$ & $C_{1 \varepsilon}$ & $C_{2 \varepsilon}$ & $C_{3 \varepsilon}$ & $C_{4 \varepsilon}$ & $C_{\mu}$ \\
\hline 0.77 & 1.44 & 1.92 & 1.2 & 1.0 & 0.09 \\
\hline
\end{tabular}

\subsubsection{Schmidt number}

The fluid turbulence also affect the drag force acting on the sediment particles. In the model description of Chauchat et al. [2017], the Schmidt number $S_{c}$ present in the two-phase flow momentum and turbulent equations (Eqns. 3, 4, 11 and 14) is a constant. However, Van Rijn [1984], based on Coleman [1970] experimental data, proposed a relationship between the Schmidt number and the ratio of the fall velocity $\left(w_{\text {fallo }}\right)$ to the bed friction velocity $u_{*}$, the so-called suspension number [Sumer et al., 1996]. In the present work, an original local Schmidt number definition for the two-phase flow model inspired from Jha and Bombardelli [2009] is used:

$$
S_{c}=\min \left[\left(1+2\left(\frac{w_{\text {fallo }}}{u_{*}+u_{\text {small }}}\right)^{2}\right)^{-1}, \frac{1}{3}\right],
$$

where $u_{\text {small }}=10^{-10} \mathrm{~m} \cdot \mathrm{s}^{-1}$ is a regularization parameter that is introduced to avoid singularity.

\subsection{Granular stresses}

The particle phase stress tensor can be split into the normal and off-diagonal components corresponding to the particle pressure $\tilde{p}^{s}$ and the particle shear stress $\tau_{i j}^{s}$, respectively. As established by Johnson and Jackson [1987] the particle normal stresses (or pressure) can be generally classified into two contributions: a shear induced or collisional component (super-script ' $s$ ') and a permanent contact component (super-script ' $\mathrm{ff}$ '):

$$
\tilde{p}^{s}=p^{f f}+p^{s}
$$

where the permanent contact component $p^{f f}$ is calculated as:

$$
p^{f f}=\left\{\begin{array}{r}
0, \phi<\phi_{\min }^{F r i c} \\
F r \frac{\left(\phi-\phi_{\min }^{F r i c}\right)^{\eta_{0}}}{\left(\phi_{\max }-\phi\right)^{\eta_{1}}}, \phi \geq \phi_{\min }^{F r i c},
\end{array}\right.
$$

with $\phi_{\min }^{F r i c}=0.57, \phi_{\max }=0.635$ for spheres. $F r, \eta_{0}$ and $\eta_{1}$ are empirical coefficients. Following Cheng et al. [2017] the values are set to: $F r=0.05, \eta_{0}=3$ and $\eta_{1}=5$. The permanent contact component is due to enduring contact in highly concentrated region that are often close to quasi-static/immobile bed. This normal pressure increases rapidly when the sediment concentration is close to maximum packing limit, and prevents unphysical sediment concentration in the sediment bed.

The second term in Eqn. (25) accounts for the shear-induced particle normal stress. The approach chosen for shear-induced particle normal stress and shear stress modelling is the dense granular flow rheology or the so-called $\mu(I)$ rheology [GDRmidi, 2004; Forterre and Pouliquen, 2008]. This approach is phenomenological, and based on dimensional analysis. It has successfully been used by Revil-Baudard and Chauchat [2013] and Chauchat [2018] to model turbulent sheet flows for instance. The total particle phase shear stress reads:

$$
\tau_{i j}^{s}=R_{i j}^{s}+r_{i j}^{s}
$$


in which $R_{i j}^{s}$ represents a Reynolds stress like contribution for the solid phase and $r_{i j}^{s}$ is the granular stress contribution coming from particle-particle interactions. The Reynolds stress contribution is modeled according to Rusche [2003] using the $C_{t}$ model. $C_{t}$ is defined as the ratio of particle rms velocity fluctuations to the fluid rms velocity fluctuations. This leads to the following Reynolds stress contribution for the particle phase:

$$
R_{i j}^{s}=\rho^{s} \phi\left[C_{t}^{2} v_{t}^{f} S_{i j}^{s}-\frac{2}{3} C_{t}^{2} k \delta_{i j}\right],
$$

According to Rusche [2003] $C_{t}$ depends on the local value of the particle concentration and varies between 1 and 3. In the present model a value of 1 has been chosen. Based on comparison with sheet-flow cases (not shown here but see Nagel [2018]), the Reynolds Stress like contribution to the particle phase momentum balance seems to be needed to better predict the suspended load. The granular contribution is written as:

$$
r_{i j}^{s}=v_{F r}^{s} S_{i j}^{s},
$$

where $S_{i j}^{s}$ is the deviatoric part of sediment phase strain rate tensor

$$
S_{i j}^{s}=\frac{1}{2}\left(\frac{\partial u_{i}^{s}}{\partial x_{j}}+\frac{\partial u_{j}^{s}}{\partial x_{i}}\right)-\frac{2}{3} \frac{\partial u_{k}^{s}}{\partial x_{k}} \delta_{i j} .
$$

In Eqn. (29), the frictional viscosity $v_{F r}^{s}$ is defined following Chauchat and Médale [2014]:

$$
v_{F r}^{s}=\min \left(\frac{\mu(I) \tilde{p}^{s}}{\rho^{s}\left(\left\|\boldsymbol{S}^{s}\right\|^{2}+D_{\text {small }}^{2}\right)^{1 / 2}}, v_{\max }\right),
$$

in which $v_{\max }$ is the maximum solid phase viscosity, $\left\|S^{s}\right\|$ is the norm of the shear rate tensor and $D_{\text {small }}=10^{-6} s^{-1}$ is a regularization parameter that is introduced to avoid singularity. In addition to the viscosity regularization, $v_{F r}^{s}$ is also clipped by $v_{\max }$ for numerical stability.

The frictional shear viscosity $v_{F r}^{s}$ allows to relate the total particle phase shear stress to the total particle pressure $\tilde{p}^{s}$ by a dynamic friction coefficient $\mu$ [Jop et al., 2006] depending on the dimensionless number I. In the present study, the regime of the granular flow rheology is in the free fall or grain inertia regime. In this regime, the friction coefficient depends on the inertial number $I=\left\|\nabla \boldsymbol{u}^{s}\right\| d \sqrt{\rho^{s} / \tilde{p}^{s}}$ according to:

$$
\mu(I)=\mu_{s}+\frac{\mu_{2}-\mu_{s}}{I_{0} / I+1}
$$

with $\mu_{s}$ the static friction coefficient, $\mu_{2}$ an empirical dynamical coefficient and $I_{0}$ an empirical constant of the rheology.

The shear induced contribution to the particle pressure can be obtained from the dilatancy law $\phi(I)$ as proposed by Boyer et al. [2011] for the viscous regime of the granular flow rheology. The adaptation to the inertial regime leads to the expression suggested by Maurin et al. [2016]:

$$
\phi(I)=\frac{\phi_{\max }}{1+B_{\phi} I},
$$


where $B_{\phi}=2 / 3$ [Chauchat, 2018].

Inverting Eqn. (33) and substituting the definition of the inertial number $I$ gives the following expression for the shear induced pressure:

$$
p^{s}=\left(\frac{B_{\phi} \phi}{\phi_{\max }-\phi}\right)^{2} \rho^{s} d\left\|\boldsymbol{S}^{s}\right\|^{2} .
$$

Finally, the total particle pressure $\tilde{p}^{s}$ can be calculated by Eqn. (25).

\subsection{Numerical implementation}

The numerical implementation of the present version of the Eulerian two-phase flow sediment transport model sedFoam is based on the open-source finite volume CFD library called OpenFOAM. The numerical implementation is similar that the one described in Chauchat et al. [2017]. The only difference lies in the pressure solver used. In the present numerical implementation, the pressure solver iterate on the reduced pressure $p^{*}$ :

$$
p^{*}=p-\rho^{f} g h,
$$

where $\mathrm{p}$ is the total pressure and $\rho^{f} g h$ is the hydrostatic pressure. This way of solving the pressure system is similar to what is done in other validated OpenFOAM solvers such as interFoam [Deshpande et al., 2012]. It has also been found that this solver is more stable compared to the one described in Chauchat et al. [2017], allowing to increase the CFL condition and reducing the computational time.

\section{Unidirectional sediment transport simulations}

Before considering the 3D scour problem, the first step is to evaluate the capability of the two-phase flow model to reproduce a unidirectional sediment transport. The objective of these simulations is to (i) evaluate the sensitivity of the results to the numerical parameter $v_{\max }$, (ii) provide a general definition of the fluid bed shear stress valid for complex geometries and (iii) verify the capability of the two-phase flow model to reproduce sediment transport rate formulas and establish a reference for the sediment transport law in undisturbed flow conditions, i.e. far from any obstacle. This reference will be used for the evaluation of the sediment transport law modification induced by the presence of the obstacle in Section 6 of the paper.

The cases are two-dimensional, the flow being restricted to a vertical plane with a dominating streamwise velocity. In order to drive the flow, an external body force or a stream-wise pressure gradient is imposed. The water depth $h_{f}=0.2 \mathrm{~m}$ and the initial sediment layer thickness $h_{s}=0.1 \mathrm{~m}$ are fixed following the Live-Bed configuration of Roulund et al. [2005]. The sediments are made of medium sand with density $\rho^{s}=2650 \mathrm{~kg} . \mathrm{m}^{-3}$ and mean grain size diameter $\mathrm{d}=0.26 \mathrm{~mm}$. The corresponding fall velocity of an individual grain in quiescent water is $w_{\text {fall }}=3.4 \mathrm{~cm} / \mathrm{s}$. The fluid is water with density $\rho^{f}=1000 \mathrm{~kg} \cdot \mathrm{m}^{-3}$ and kinematic viscosity $v^{f}=10^{-6} \mathrm{~m}^{2} \cdot \mathrm{s}^{-1}$. The mean fluid flow velocity is $\bar{U}=0.46 \mathrm{~m} \cdot \mathrm{s}^{-1}$. The initial concentration profile is imposed using a hyperbolic tangent profile. The stream-wise pressure gradient is computed from the bed friction velocity of $u_{*}=2.8 \mathrm{~cm} / \mathrm{s}$ estimated from the experiments by Roulund et al. [2005]:

$$
f_{p x}=\frac{\rho^{f} u_{*}^{2}}{h_{f}}
$$


The Shields parameter at the inlet is the same as is in Roulund et al. [2005], $\theta=0.19$. It can be seen as the ratio between the destabilizing (fluid shear stress, $\tau_{b}$ ) and stabilizing forces (grains weight) acting on a particle:

$$
\theta=\frac{\tau_{b}}{\left(\rho^{s}-\rho^{f}\right) g d}
$$

The water column is discretized using 64 vertical levels with a geometric common ratio $\mathrm{r}_{f}=1.075$ (from the initial interface to the top). In the sediment bed, 50 vertical levels with a geometric common ratio $r_{s}=1.086$ (from the initial interface to the bottom) are used. The vertical profiles of this configuration will be used as inlet boundary conditions in the $3 \mathrm{D}$ simulation presented in Section 4.

The granular rheology parameters are set up as follows: the static friction coefficient is set to $\mu_{s}=0.63$ corresponding to the tangent of the angle of repose for sand in water, the dynamical friction coefficient is fixed to $\mu_{2}=1.13$ and $I_{0}=0.6$. These values have been obtained for bedload transport with spheres using the Discrete Element Method [Maurin et al., 2016]. In order to account for the particle shape, the coefficients $\mu_{s}$ and $\mu_{2}$ have been increased by a constant value so that $\mu_{s}$ matches the tangent of the repose angle for real sand particles $\beta_{r}=32^{\circ}$.

A sensitivity analysis to the solid phase maximum viscosity, $v_{\text {Max }}$ has been performed in Nagel [2018]. The model shows a strong sensitivity at low values of $v_{\text {Max }}$ but converges to a constant value when $v_{\text {Max }}$ increases. The error associated with $v_{\text {Max }}$ is called the "creeping flow", if the maximum viscosity is too low a non negligible velocity gradient will be predicted in the "static sediment bed" that can give rise to a non-negligible spurious sediment transport flux. The creeping flux decays exponentially as $v_{\text {Max }}$ increases. It has been shown that a value of $v_{\text {Max }}=100 \mathrm{~m}^{2} / \mathrm{s}$ should be used to guarantee a negligible creeping flow.

By varying the pressure gradient, we will investigate the sediment transport flux as a function of the Shields parameter. The dimensionless depth integrated sediment flux, $q^{*}$ and the dimensionless transport layer thickness $\delta^{*}$ are computed from the numerical solutions and plotted against the Shields parameter $\theta$ in Figure 1.

The dimensionless depth integrated sediment flux is calculated as the total sediment transport rate including the contribution of the bed-load and the suspended-load:

$$
q^{*}=\frac{\int \boldsymbol{u}^{s} \phi \mathrm{d} z}{\sqrt{(s-1) g d^{3}}} .
$$

The dimensionless transport layer thickness is computed as the bed-normal distance between the iso-surfaces $\phi=0.57$ and $\phi=0.08$ made dimensionless by the particle diameter $d$ :

$$
\delta^{*}=\delta / d,
$$

s where $\phi=0.57$ is a proxy for the immobile bed position and $\phi=0.08$ corresponds to a an inter-particle distance of one particle diameter which is taken as the transition between bedload and suspended load layers [Bagnold, 1956; Dohmen-Janssen et al., 2001]. In addition to Roulund et al. [2005] unidirectional bedload case, $q^{*}$ and $\delta^{*}$ are also calculated from simulation undertaken with Revil-Baudard et al. [2015] and Sumer et al. [1996] unidirectional sheet-flow configurations (described in Chauchat et al. [2017]). These three configurations cover a wide range of Shields number $\theta \in[0.1 ; 2.5]$ for different particle sizes and densities (see Table 3).

In the left panel of Figure 1, the dimensionless sediment transport rate is plotted against the Shields number. For the three configurations investigated the numerical results are within the 
Table 3: Shields, particle sizes and particle densities for the unidirectional sediment transport configuration.

\begin{tabular}{cccc}
\hline Case & Shields & Particle size $(\mathrm{mm})$ & Particle density ratio $s$ \\
\hline Roulund et al. [2005] & {$[0.1 ; 0.7]$} & 0.26 & 2.65 \\
Revil-Baudard et al. [2015] & 0.6 & 3 & 1.19 \\
Sumer et al. [1996] & {$[1.5 ; 2.5]$} & 2.6 & 1.14 \\
\hline
\end{tabular}

scatter of the experimental data, namely Meyer-Peter and Müller [1948] for $\theta \leq 0.5$ and Wilson [1966] for $\theta \geq 0.5$.

In the right panel of Figure 1 the dimensionless sediment transport layer thickness is plotted against the Shields number. For Revil-Baudard et al. [2015] and Roulund et al. [2005] configurations the results exactly match Wilson [1987] semi-empirical model $\left(\delta^{*}=10 \theta\right)$. The results for Sumer et al. [1996] case does not exactly follow that law but the trend is similar and the numerical results are in the scatter of Sumer et al. [1996] experiments.
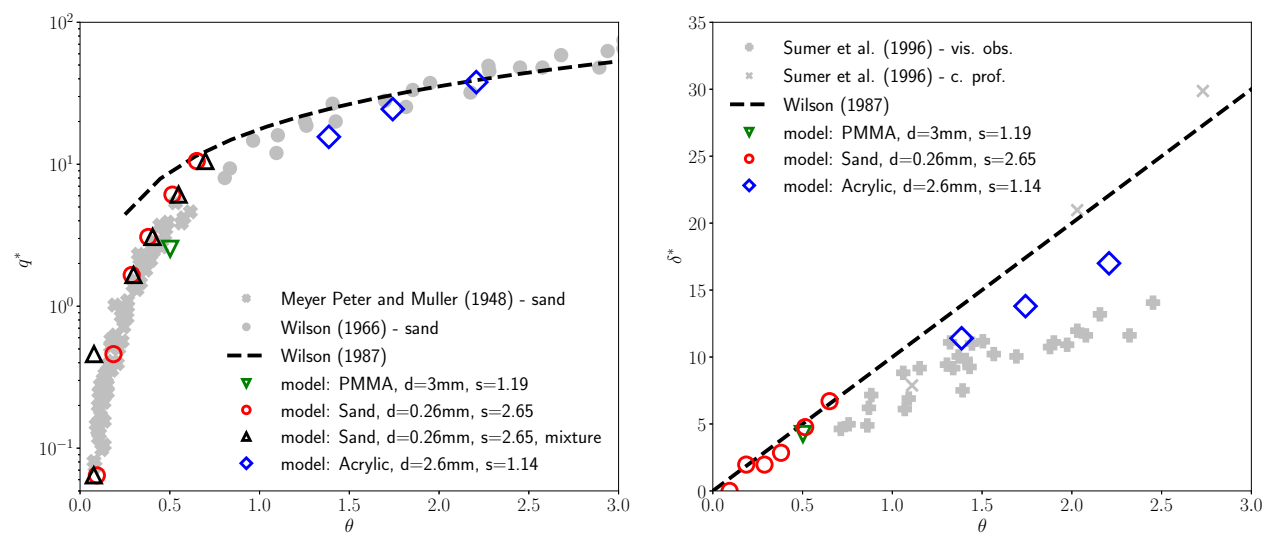

Fig. 1: Dimensionless sediment transport rate $q^{*}$ (left) and dimensionless sheet layer thickness $\delta^{*}=\delta / d$ (right) versus the Shields number predicted by SedFoam: red circles and dark triangles correspond to Roulund et al. [2005] unidirectional bedload configuration, green triangles and blue diamonds correspond to Sumer et al. [1996] and Revil-Baudard et al [2015] unidirectional sheet flow configuration, respectively.

The definition of the bed shear stress used to evaluate the Shields number is also a matter of debate. In Chauchat [2018] the maximum value of the fluid bed shear stress is used:

$$
\tau_{b}=\max \left(R_{x z}^{f}\right)
$$

This definition corresponds to the red empty circles plotted in the left panel of Figure 1. In a unidirectional case, computing the bed shear stress as the maximum of the fluid shear stress lead to a very good agreement with literature data (see Figure A4 of Chauchat [2018]). However, when dealing with complex 3D flow configurations this definition may lead to an inconsistency as the fluid shear stress profile does not necessarily present a monotonous increase toward the bed. We propose an alternative, consisting of the use of the mixture shear stress computed at the elevation of the iso-concentration $\phi_{0}=0.08$ to compute the Shields number:

$$
\tau_{b}=\left.\tau_{x z}^{f}\right|_{\phi=\phi_{0}}+\left.\tau_{x z}^{s}\right|_{\phi=\phi_{0}}
$$


A sensitivity analysis to $\phi_{0}(0.08,0.45,0.57)$ has been performed and the Shields number estimation is only marginally affected (not shown here, see Nagel [2018] for details). For clarity, only the results obtained using $\phi_{0}=0.08$ are plotted with the dark empty triangles in the left panel of Figure 1.

A sensible choice for the bed shear stress definition would be to consider the actual shear stress exerted by the mixture of grains and fluid at the fixed bed interface. This corresponds to the latest definition using $\phi_{0}=0.57$ and works well for unidirectional steady uniform flows. However, when the flow is non uniform with steep bed slopes it is more complex to relate the Shields number to the actual shear stress exerted on the immobile bed as other granular processes are involved. It should be noted here that the Shields number characterizes the fluid drag force exerted by the flow on the particles in the near bed region. In the perspective of upscaling the two-phase flow results in single-phase sediment transport models, it is important to use a resolved quantity in the single-phase flow model to compute the Shields number. The shear stress in the dilute region of the flow is solved by the single-phase flow model. However, the shear stress exerted by the grain-fluid mixture on the immobile bed is not.

In order to establish the undisturbed reference solution against which a $3 \mathrm{D}$ simulation can be compared, the one-dimensional results for medium sand in the range of Shields parameter between 0.1 to 2.5 can be fitted using a power law of the excess Shields number:

$$
q_{\text {mod }}^{*}=a\left(\theta-\theta_{c}\right)^{b},
$$

where $\theta_{c}=0.047$ is the critical Shields number. The $a$ and $b$ coefficients obtained from the best fit are summarized in Table 4. First of all, the values obtained are consistent between the different methods for the bed shear stress estimation. The exponent of the power law $b$ is less sensitive and in better agreement with classical empirical formula of Meyer-Peter and Müller [1948] or Wong and Parker [2006]. The prefactor $a$ is more sensitive with values ranging between 25 and 32 which is quite far from the empirical values of 3.97 and 8 . It should be noted that all the different cases have different suspension numbers. According to Sumer et al. [1996], the suspension number can be written as $S_{u}=w_{f a l l 0} / u_{*}$. For $S_{u}<1$ (corresponding here to $\theta>0.3$ ), the suspension becomes important in the total sediment flux. For the sand case, $S_{u}$ varies between 1.6 (for $\theta=0.1$ ) and 0.56 (for $\theta=1$ ). Therefore, a non-negligible suspended-load components to the total sediment flux is expected. In these conditions it is not surprising that the coefficients $a$ and $b$ are different from the values of Meyer-Peter and Müller [1948]. Nevertheless, the model is able to recover a power law, with a reasonable exponent. It is worth mentioning that an exponent $b=2$ has been obtained in all former studies using two-phase flow models [Hsu et al., 2004; Revil-Baudard and Chauchat, 2013; Chauchat, 2018]. Interestingly, when using the mixture stress at sediment concentration higher than 0.08 , the exponent $b$ tends toward 1.5 consistently with Meyer-Peter and Müller [1948] or Wong and Parker [2006]. It is therefore kept as the definition of the fluid bed shear stress to analyze the 3D scour configuration.

\section{3D Scour simulation}

When an obstacle, such as a bridge pier or a river restoration structure, is built in a stream, its presence can strongly modify the flow field and induce scour. For example, around a cylindrical pier, the flow separation creates a strong adverse pressure gradient. As a result, the flow plunges toward the bed and if the bottom boundary layer contains a sufficient amount of vorticity, a horseshoe vortex (HSV) is generated at the bed-structure junction. Along the sides of a 
Table 4: Estimated value of the power law coefficients of the excess Shields parameter.

\begin{tabular}{cccc}
\hline Bed shear stress estimation & Concentration $\left(\phi_{0}\right)$ & $a$ & $b$ \\
\hline Meyer-Peter and Müller [1948] & - & 8.0 & 1.50 \\
Wong and Parker [2006] & - & 3.97 & 1.50 \\
eq. 40 & - & 29.9 & 2.07 \\
eq. 41 & 0.45 & 31.06 & 1.57 \\
eq. 41 & 0.3 & 28.56 & 1.59 \\
eq. 41 & 0.08 & 26.14 & 2.09 \\
\hline
\end{tabular}

structure, streamline contraction leads to flow acceleration and amplify the bed shear stress. At the downstream side of the structure, the boundary layer can separate and lead to vortex shedding in the wake. In combination, the wake vortices and the HSV lead to a local increase of bed shear stress, sediment transport rate and bed erosion. Scour hole development, if not correctly predicted and treated, may significantly undermine the structure and cause its failure with potentially disastrous consequences [Breusers et al., 1977; Dargahi, 1990; Breusers and Raudkivi, 1991; Melville and Coleman, 2000; Roulund et al., 2005].

In the previous section, it has been demonstrated that the two-phase flow model presented in Section 2 is able to accurately predict unidirectional sediment transport for medium sand. The range of Shields number investigated corresponds to the expected range in the 3D scour configuration of Roulund et al. [2005]. Furthermore, the detailed study of the hydrodynamics around a vertical cylinder in a steady current without sediments is presented in Appendix A. It ensure that sedFoam is able to accurately reproduce the flow key structures, i.e the HSV and the lee-wake vortices, for the scour phenomenon. In the present section, the two-phase flow model will be used to investigate the more complex configuration of the scour around a cylindrical pile.

\subsection{Numerical configuration}

The numerical domain can be divided into two parts: the initial water domain and the initial sediment domain (grey part in Figure 2). The initial water domain is a three-dimensional box with a stream-wise length $L x=13 \mathrm{D}$, a span-wise length $\mathrm{Ly}=8 \mathrm{D}$ and a height $\mathrm{H}=2 \mathrm{D}$ where $\mathrm{D}=10 \mathrm{~cm}$ is the pile diameter, as in Roulund et al. [2005]. At the bottom, a thin sediments layer (0.25D) extends below the entire water domain except in a region around the pile, where a deeper circular pit of height $H_{s}=\mathrm{D}$ and radius $r_{p i t}=2 \mathrm{D}$ has been setup to reduce the grid cells number. The sediments properties are the same as in the unidirectional case (see section 3). The Shields parameter at the inlet and the Reynolds number are the same as is in Roulund et al. [2005], $\theta=0.19$ and $\operatorname{Re}_{D}=4.6 \times 10^{4}$, respectively.

The computational domain is discretized using a unstructured mesh (see Table 5). The mesh is refined around the cylinder and at the initial interface position. The mesh refinement area around the cylinder is axisymmetric. As for the unidirectional case, the water column is discretized using 64 vertical levels with a geometric common ratio $\mathrm{r}_{f}=1.075$ (from the initial interface to the top).

For the sand layer, outside the scour pit, the mesh is composed of 100 vertical levels having a geometric distribution with a common ratio $\mathrm{r}_{s}=1.025$. In the pit, an additional 100 grid points are used with a geometric common ratio $\mathrm{r}_{p i t}=1.010$. 


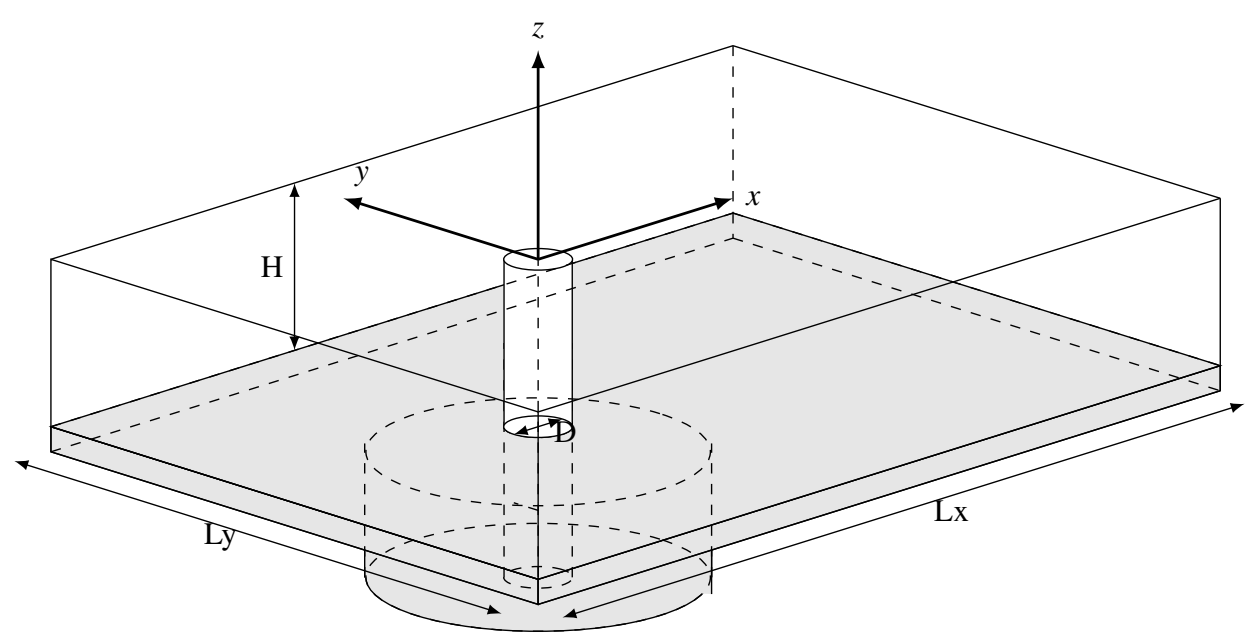

Fig. 2: Sketch of the geometry (illustration is adapted from Roulund et al. [2005]).

Table 5: Summary of the geometry and mesh characteristics.

\begin{tabular}{cc}
\hline Characteristics & \\
Lx & $13 \mathrm{D}$ \\
Ly & $8 \mathrm{D}$ \\
Total number of cells & 5308368 \\
Number of cells around the cylinder perimeter & 256 \\
Horizontal resolution around the cylinder perimeter (m) & $1.2 \times 10^{-3}$ \\
Number of cells across the water depth & 64 \\
Number of cells across the sediments & 200 \\
Number of cells across the scour Pit & 100 \\
Initial interface cell height (m) & $1.5 \times 10^{-4}$ \\
\hline
\end{tabular}

The boundary conditions are imposed as follows:

(i) At the inlet, vertical profiles obtained from the unidirectional simulation are imposed for $\boldsymbol{u}^{s}$, $\boldsymbol{u}^{f}, \mathrm{k}, \omega, \phi$. Zero transverse velocity is prescribed.

(ii) At the outlet, zero-gradient conditions (Neumann conditions, $\partial / \partial_{n}=0$ ) are specified for all quantities, except for the reduced pressure for which a homogeneous Dirichlet boundary condition is imposed $\left(p^{*}=0\right)$. For the velocities, a homogeneous Neumann boundary condition is used when the velocity vector points outside of the domain at the outlet, and a homogeneous Dirichlet boundary condition $\left(\boldsymbol{u}^{\boldsymbol{k}}=0\right)$ is used otherwise.

(iii) At the top boundary, Neumann boudary conditions are applied for $\mathrm{k}, \omega$ and $\boldsymbol{u}^{k}$.

(iv) At the walls (including the cylinder), zero velocity (no-slip) is imposed for the three components and a small value is imposed for the TKE. The boundary condition for $\omega$ is specified using the classical wall function from OpenFOAM at the cylinder and a constant value is imposed at the rigid bottom. 
(v) At the side, cyclic conditions are used.

For the initial condition, the unidirectional solution used for the inlet is imposed over the entire numerical domain. Finally, the granular rheology parameters used are the same as for the unidirectional sediment transport case.

The scheme used for the divergence operators of the different quantities is a blend between a pure centered second order scheme and a first order upwind scheme in the regions of rapidly changing gradient ("limitedLinear 1"). The laplacian scheme for all quantities is a linear interpolation with non-orthogonality correction ("Gauss linear corrected").

As for the unidirectional configuration, the bed shear stress is computed as the mixture shear stress at the concentration $\phi_{0}=0.08$ :

$$
\left|\tau_{b}\right|=\sqrt{\left.\tau_{x z}^{f}\right|_{\phi=\phi_{0}}{ }^{2}+\left.\tau_{y z}^{f}\right|_{\phi=\phi_{0}}{ }^{2}+\left.\tau_{x z}^{s}\right|_{\phi=\phi_{0}}{ }^{2}+\left.\tau_{y z}^{s}\right|_{\phi=\phi_{0}}{ }^{2}} .
$$

Because the scour is deforming the initial flat sediment bed, the resulting interface can be inclined and these variations have to be accounted for in the bed shear stress calculation which is taken as the magnitude of the tangential shear stress with respect to the sediment bed surface. The bed interface is first interpolated from the original unstructured grid to a cartesian one. On each cell of the cartesian grid, the normal $\vec{n})$ and the tangential $\left(\overrightarrow{t_{x}}\right.$ and $\left.\overrightarrow{t_{y}}\right)$ vectors are calculated using the bed interface elevation horizontal gradient. The projection of the mixture bed shear stress magnitude on the plane tangential to the local bed surface reads:

$$
\left|\tau_{b}\right|=\sqrt{\left(\overrightarrow{T^{f}} \cdot \overrightarrow{t_{x}}\right)^{2}+\left(\overrightarrow{T^{f}} \cdot \overrightarrow{t_{y}}\right)^{2}+\left(\overrightarrow{T^{s}} \cdot \overrightarrow{t_{x}}\right)^{2}+\left(\overrightarrow{T^{s}} \cdot \overrightarrow{t_{y}}\right)^{2}}
$$

where $T^{k}$ is the stress vector applied on the sediments bed surface of the phase $k$. It is obtained from the product between the phase shear stress tensor $\tau^{k}$ and the vector normal to the isosurface $\phi_{0}=0.08, \vec{n}$ :

$$
\overrightarrow{T^{k}}=\tau^{k} \cdot \vec{n}
$$

For more details about the specific methodology for the bed shear stress determination in a nonuniform three-dimensional case, the interested reader is referred to Nagel [2018].

In the following, the results of the three-dimensional two-phase flow simulations are presented. The reference two-phase flow simulation was run for 600 s of real time. This computation took approximately 480 hours (20 days) on 224 processors Intel ${ }^{\circledR}$ Xeon ${ }^{\circledR}$ E5-2690 v4. The computational time is approximatively of 108,000 CPU hours, which would correspond roughly to 12 years on a single processor.

\subsection{Erosion pattern and maximum erosion depth}

In order to qualitatively illustrate the model results, snapshots of dimensionless bed elevation $(S / D)$ and fluid velocity streamlines are shown at different times $(\mathrm{t}=10 \mathrm{~s}, 60 \mathrm{~s}, 150 \mathrm{~s}$ and $600 \mathrm{~s})$ in Figure 3. The bed interface is defined as the surface of iso-concentration $\phi=0.57$. These results can be qualitatively compared with Figure 33 from Roulund et al. [2005]. It is noted that the vortex-shedding and the suspension-load are not resolved in Roulund et al. [2005]'s steadystate flow simulations. Despite these differences, both models exhibit the following bathymetric 
features: (i) a semi-circular shaped scour mark is predicted at the upstream side of the pile, (ii) sediments eroded from the scour mark first accumulate downstream the pile (see Figure 3.a), (iii) at later stages a scour mark is predicted at the downstream side (see Figures 3.b, 3.c and 3.d). This comparison supports, at least qualitatively, the relevance of the two-phase flow approach for modeling scour around hydraulic structures. The velocity streamlines show that the scour hole erosion modify the HSV but also that vortex-shedding is taking place at the downstream side of the pile.

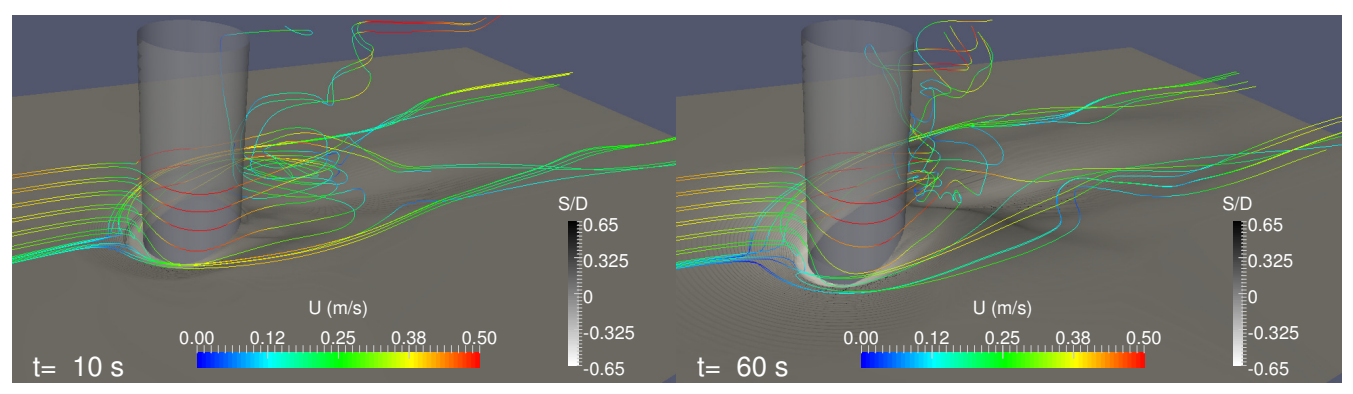

(a)

(b)

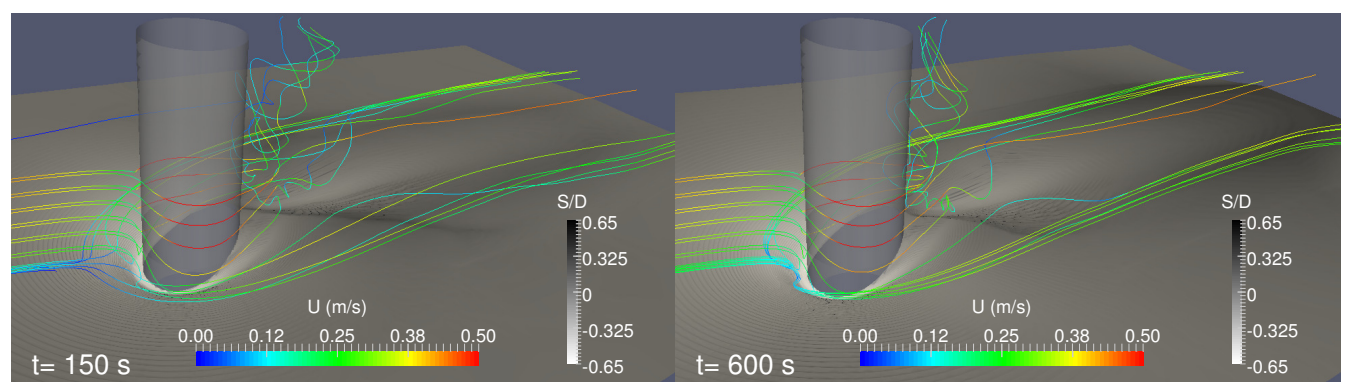

(c)

(d)

Fig. 3: Bed elevation after 10s (a), 60s (b), 300s (c) and 600s (d) of dynamics.

More quantitatively, Figure 4 shows the time evolution of the maximum dimensionless scour depth $-S / D$ at the upstream side (top panel) and at the downstream side (bottom panel) of the pile. The results of two different two-phase flow turbulence models, the $k-\omega 2006$ (blue line) and the $k-\varepsilon$ (yellow lline) are shown. They are compared with experimental data (red dots) and single-phase flow model results from Roulund et al. [2005] (green dashed line) and Stahlmann et al. [2013] (magenta dashed line).

The $k-\omega 2006$ simulation has been performed up to 600s of dynamics. The good agreement between this two-phase flow simulation and the experiments at the upstream side of the pile shows that the two-phase flow model is able to accurately reproduce the upstream scour depth evolution up to 300 s. From 300 to 600 s the maximum dimensionless scour depth is underestimated compared with the experimental data. As a result, at $t=600 \mathrm{~s}$, the two-phase flow results give $-S / D=0.63$ whereas Roulund et al. [2005] measured results show $-S / D=0.8$. 
The $k-\varepsilon$ simulation has been run for 350s. The results in terms of upstream scour are almost identical to the $k-\omega 2006$ up to 150 s. Beyond that time, the results follow a logarithmic growth and are closer to the ones from Roulund et al. [2005] using a classical sediment transport model. The two-phase flow simulation results are sensitive to the turbulence model. This confirms the simulation results of Mathieu et al. (2019) for the scour around a pipeline: using $k-\varepsilon$ model leads to an overestimation whereas using $k-\omega 2006$ model leads to an underestimation of the scour depth. However, it is important to notice that, both, the experimental results of Roulund et al. [2005] and their best numerical prediction using a classical model (Stahlmann et al. [2013]) are within the range of the solutions given by the two-phase flow model.
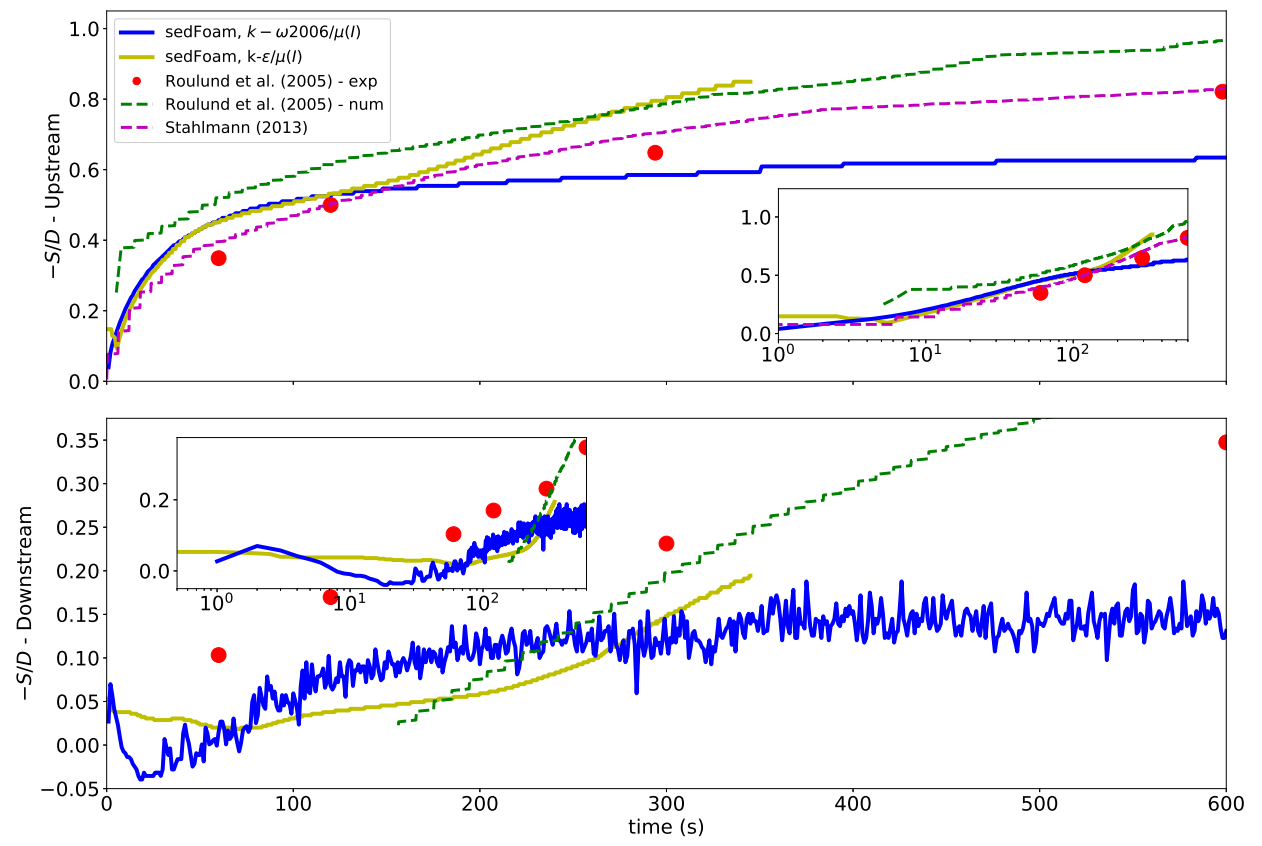

Fig. 4: Time evolution up to $600 \mathrm{~s}$ (10min) of the dimensionless scour depth at the upstream (top panel) and at the downstream (bottom panel) edge of pile.

At the downstream side (bottom panel of Figure 4) the maximum erosion depth predicted by the $k-\omega 2006$ model exhibits temporal fluctuations whereas the $k-\varepsilon$ model results exhibit a smooth curve. Unlike the upstream side, the position of the maximum scour depth downstream of the cylinder is located away from the structure at about 1 to 2 cylinder diameter downstream. The fluctuations, which are not present in Roulund et al. [2005] steady state numerical results, are most probably due to the eddies shed downstream of the pile. This result indicates that the downstream scour is highly influenced by the vortex-shedding, a result that has already been pointed out in former studies using classical sediments transport models [e.g. Zhao et al., 2010; Stahlmann et al., 2013; Baykal et al., 2015]. However, if the maximum erosion depth predicted by the $k-\omega 2006$ model follow a tendency similar to the experimental results from Roulund et al. [2005], the erosion rate is underestimated by the two-phase flow model. This leads to an 
underestimation of the erosion depth downstream of the pile.

As for the upstream side, the $k-\varepsilon$ scour depth results are very similar to Roulund et al. [2005] classical model results. No fluctuations are observed, meaning that the two-phase $k-\varepsilon$ model fails to reproduce the vortex-shedding.

\subsection{Choice of the granular stress and turbulence model}

The first point that needs to be discussed is the choice of the granular stress model. One could argue that the kinetic theory of granular flows [Jenkins and Savage, 1983; Ding and Gidaspow, 1990; Jenkins and Hanes, 1998] shall be preferred to predict sediment transport in scour configuration. Indeed, it describes better the intermediate concentration region $(0.08<\phi<0.3)$ where collisional interactions dominates. However, the $\mu(I)$ rheology reproduces more accurately the dense granular flow regime $(0.3<\phi<0.6)$ where frictional interactions dominates. For an unidirectional sheet flows case, Chauchat et al. [2017] have demonstrated that the vertical structure of the flow as well as the repartition of the sediment flux is only slightly affected by the choice of the granular stress model. Furthermore, Mathieu et al. [2019] recently performed a sensitivity analysis to the choice of the granular stress model using the same numerical model for the scour below a pipeline configuration. The authors observed almost no sensitivity to the choice of the granular stress model on the erosion depth. Due to the very high computational cost of the 3D scour simulations this sensitivity analysis is not performed herein.

Unlike classical sediment transport models, two-phase flow models are process-based models and as such shall not be fine-tuned on each configuration. For the $\mu(I)$ rheology, the parameters have been calibrated on unidirectional sediment transport configurations in Section 3. The unicity and accuracy of this calibration could be questionned, however, for sake of consistency it has been decided not to modify these paremeters in the 3D simulations. In classical sediment transport models, the sediment transport fluxes are based on emprirical formula obtained under the assumptions of unidirectional steady and uniform flows. Applying these parametrizations to non-uniform and unsteady flow conditions raises potential inconsistencies and a sensitivity analysis to some of the empirical constant is usually needed to improve the model predictions (e.g. Stahlmann et al. [2013]).

As already pointed out by Mathieu et al. [2019] for the scour around the pipeline, the near bed erosion is probably better described by the $k-\varepsilon$ model and the lee-wake erosion is better predicted by the $k-\omega 2006$ model. As a matter of fact, in the vertical cylinder configuration, the $k-\omega 2006$ turbulence model is the only one able to reproduce the vortex-shedding downstream of the cylinder and to account for the adverse pressure gradient upstream of it [Roulund et al., 2005]. Therefore, in the following, all the results presented are obtained with the k- $\omega 2006$ turbulence model.

\section{Analysis of the sediment fluxes}

\subsection{Upstream and around the pile}

In the case of a flow around a structure, the assumption of uniform flow condition is not verified. It has been shown in Section 3 that the two-phase flow model predicts a power law for the relationship between the sediments transport flux and the Shields parameter under uniform and steady flow conditions. In Section 4.2 it has been further demonstrated that the two-phase 
flow model is able to predict reasonably well the bed morphological evolution at the early stages of the scour process. In this section, the numerical results are further analyzed to investigate the perturbation induced by the pile on sediment transport and how much it differs from the results obtained under steady and uniform flow conditions.

Figure 5.a shows the local slope angle, $\beta$, at $\mathrm{t}=10 \mathrm{~s}$. It is determined from the magnitude of the horizontal gradient of the bed elevation:

$$
\beta=\arctan \left(\nabla_{h} z_{b e d}\right),
$$

where $\nabla_{h} z_{\text {bed }}=\sqrt{\left(\frac{\partial z_{b e d}}{\partial x}\right)^{2}+\left(\frac{\partial z_{\text {bed }}}{\partial y}\right)^{2}}$ and $z_{\text {bed }}=\left.z\right|_{\phi=0.57}$. Far from the obstacle the bed is almost flat but closer to the cylinder, there is a semi-circular area where the slope is gently increasing toward the cylinder. The upstream distance to the center of the cylinder is defined as $R_{u}$, it is equal to $\mathrm{x}$ on the $\mathrm{x}$-axis. At the upstream side of the cylinder, $R_{u} / D \approx 0.7$, an important variation of the bed slope angle is visible. $\beta$ varies between $\beta \approx 30^{\circ}$ and $\beta \approx 45^{\circ}$ exceeding the angle of repose $\left(\beta_{r}=32^{\circ}\right)$. In this region, avalanches occur and the sediment flux is predominately driven by gravitational acceleration. Closer to the cylinder, $0.6>R_{u} / D>0.5$, the slope angle is decreasing and the sediment bed is nearly flat. Just downstream of the cylinder, up to $x / D=1$, the bed slope angle is more noisy and it is hard to distinguish any slope tendency. Further downstream, $(x / D>1)$ the bed variations are very small and $\beta$ remains below $10^{\circ}$.

Figure 5.c shows the instantaneous dimensionless depth integrated sediment flux calculated by the two-dimensional extension of Eqn. (38). The sediment flux starts to increase where the bed slope angle is increasing and it peaks around $R_{u} / D \approx 0.7$. Closer to the cylinder, where the scour hole is the deepest and the bed is almost flat, the sediment flux becomes very small. The maximum of the sediment flux is located in the two HSV legs around the cylinder. This result indicates that there is an important transverse flux driven by the HSV legs around the cylinder. This is in agreement with the description of Link et al. [2012]. The maximum dimensionless sediments flux within these two legs is $q_{\max }^{*}=7.9$. Downstream of the cylinder, the sediment flux is weak, probably because the vortex-shedding is not fully developed at this early stage $(t=10 \mathrm{~s})$. The patch of sediment flux appears to be due to a vortex shed from the cylinder passing by.

Figure 5.b shows the spatial distribution of the Shields number. Its magnitude is slightly increasing with the slope in the scour hole and reaches its maximum at the sides of the cylinder, where the Shields number values correspond to sheet-flow regime $(\theta>0.3)$, i.e for $\Gamma \in$ $\left[ \pm 65^{\circ}- \pm 120^{\circ}\right] . \Gamma$ is the angle measured with respect to the upstream $x$-axis. Downstream of the pile, a high Shields number area is located around $x / D=1$ and $y / D=-0.25$, exactly where an increase of sediments transport was observed in Figure 5.c. Here, the local bed shear stress has a swirl structure confirming that it is generated by a vortex shed from the cylinder.

Figure 5.d shows the dimensionless sediments flux estimated using the power law deduced from unidirectional simulations presented in Section 3:

$$
q^{*}=a\left(\theta-\theta_{c S}\right)^{b},
$$

with $\mathrm{a}=26.14$ and $\mathrm{b}=2.09$, i.e using bottom shear stress evaluated at vertical elevation where $\phi_{0}=0.08$. The critical Shields number $\theta_{c S}$ is defined as a function of the local bed slope and 
orientation. The formulation used in the present work is same as in Roulund et al. [2005]. The flow velocity at the particle position and the steepest slope orientation with respect to the flow are used to adjust the critical Shields number:

$$
\theta_{c S}=\theta_{c}\left(\cos \beta \sqrt{1-\frac{\sin ^{2} \alpha \tan ^{2}(\tilde{\beta})}{\mu_{s}^{2}}}-\frac{\cos \alpha \sin (\tilde{\beta})}{\mu_{\mathrm{s}}}\right),
$$

where $\theta_{c}=0.047$ is the critical Shields number for a flat bed, $\tilde{\beta}=\min \left(\beta, \beta_{r}\right)$ and $\alpha$ is the angle between the near-bed velocity and the direction of maximum slope:

$$
\alpha=\arccos \left(\frac{\nabla_{\mathrm{h}} \mathrm{Z}_{\mathrm{bed}} \cdot \boldsymbol{u}^{s}\left(\phi_{\mathbf{0}}\right)}{\left\|\nabla_{\mathrm{h}} \mathrm{Z}_{\mathrm{bed}}\right\|\left\|\boldsymbol{u}^{s}\left(\phi_{0}\right)\right\|}\right),
$$

in which $\boldsymbol{u}^{s}(\phi)$ is the sediment velocity at the iso-concentration $\phi=0.08$. This concentration being the best compromise in term of bed shear stress estimation [Nagel, 2018].

Eqn. (48) has been slightly modified from Roulund et al. [2005] definition. The original definition provides non-physical values for slope angles above the angle of repose $\left(\beta_{r}=32^{\circ}\right)$. In classical sediment transport models, an avalanche module is required to prevent the bed slope to exceed the angle of repose. In the two-phase flow model, the bed slope is free to exceed that critical value while the solution of the momentum balance will predict a physical description for the avalanching process, e.g. Bagnold profile for dry granular flows [Andreotti et al., 2013].

The dimensionless sediment flux obtained using Eqn. (47) is locally correlated to the bed shear stress or the Shields number. As expected, the dimensionless sediment flux shown in Figure 5.d and the Shields number shown in Figure 5.b present the same spatial patterns: they increase in the upstream part of the scour hole, reach a maximum value for $\Gamma \in\left[ \pm 65^{\circ}- \pm 120^{\circ}\right]$, but are low for $\Gamma \in\left[0^{\circ}- \pm 45^{\circ}\right]$ and close to the cylinder. Due to vortex shedding, the instantaneous flow pattern shown in Figure 5 is asymmetric. The spatial correlation between depth integrated sediments flux as predicted by the two-phase flow model (subfigure 5.c) and by the empirical classical power law given by Eqn. (47) (subfigure 5.d) is rather poor. Indeed, for $\Gamma \in\left[0^{\circ}- \pm 65^{\circ}\right]$, and more particularly around the steep slope upstream the cylinder, the important sediment flux observed in subfigure 5.c is not observed in subfigure 5.d. This result suggests that in this region, sediment transport is not driven by the local fluid bed shear stress but rather by the bed slope.

The second and third columns of Figure 5 show the same quantities for $t=60 \mathrm{~s}$ and $\mathrm{t}=150 \mathrm{~s}$, respectively. The bed slope variations are very similar between $t=60$ and $t=150 \mathrm{~s}$. In subfigures 5.e and 5.i, the slope angle increases toward the cylinder, exceeding the angle of repose between $R_{u} / D \approx 0.7$ and $R_{u} / D \approx 0.6$. The slope starts to be important further upstream at $\mathrm{t}=150 \mathrm{~s}$. This is in good agreement with the upstream extension of the scour hole as time increases.

Between $R_{u} / D \approx 0.7$ and the pile, for $\Gamma \in\left[0^{\circ} ; \pm 45^{\circ}\right]$, the Shields number (subfigures $5 . f$ and 5.j) is below its undisturbed value of 0.19 . This is due to the presence of the cylinder, which generates the down-flow. This leads to a reduction of the Shields number in front of the cylinder. The maximum Shields number is located downstream of the cylinder and at the cylinder sides for $\Gamma \in\left[ \pm 65^{\circ} ; \pm 145^{\circ}\right]$ where it is decreasing in time. Downstream of the pile, due to the vortexshedding, the Shields number is important in a narrow channel approximatively aligned with the $\mathrm{x}$-axis.

At $\mathrm{t}=60 \mathrm{~s}$ and $\mathrm{t}=150 \mathrm{~s}$, the depth integrated dimensionless sediments flux obtained directly from the velocity and concentration fields (see subfigures 5.g and 5.k) have similarities to the 

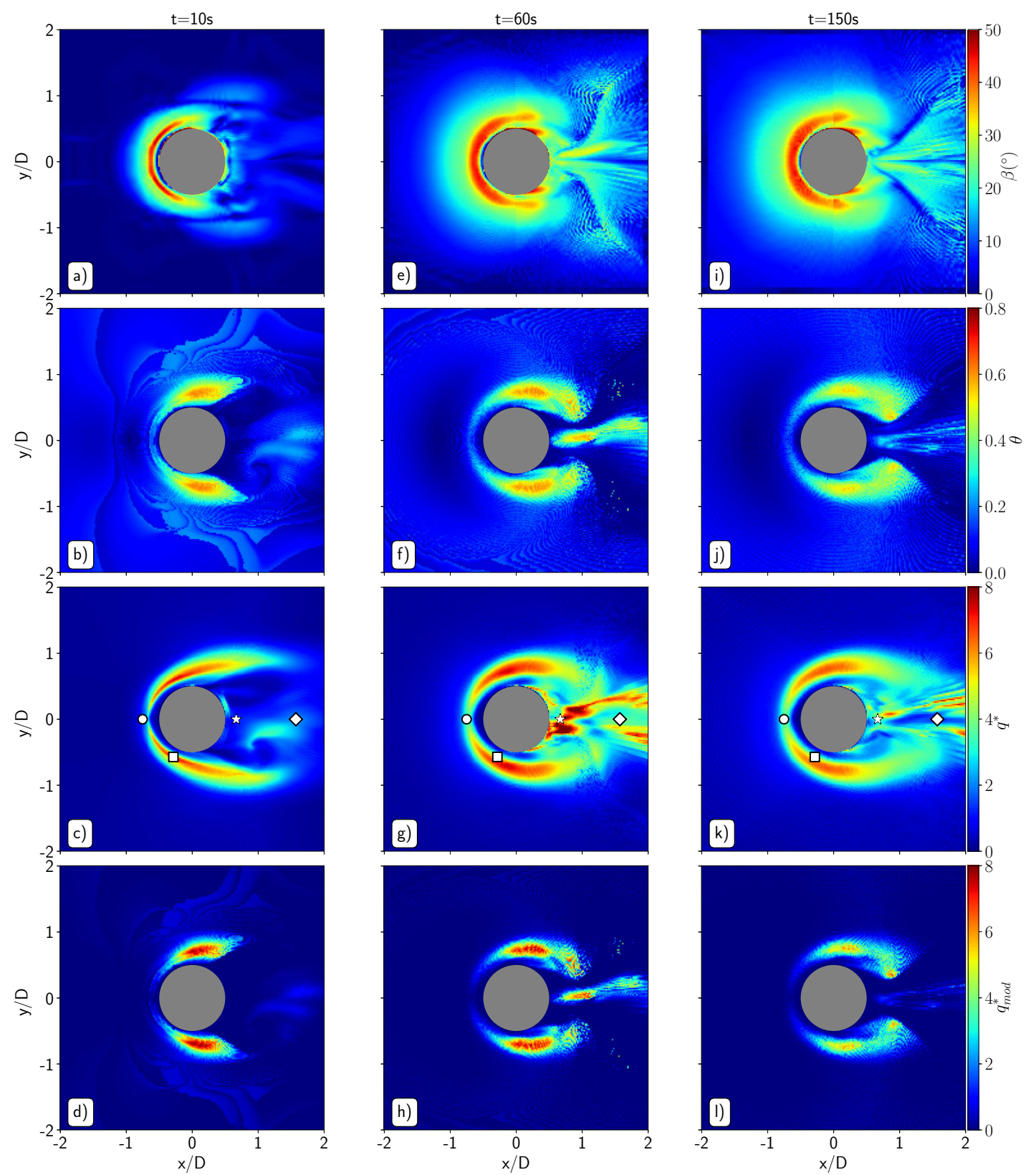

Fig. 5: Sediments bed slope angle (a,e,i), Shields number (b,f,j), dimensionless depth integrated sediments flux (c,g,k) and dimensionless sediments flux estimated from "classical" power law with slope correction $(\mathrm{d}, \mathrm{h}, \mathrm{l})$ at $\mathrm{t}=10 \mathrm{~s}, \mathrm{t}=60 \mathrm{~s}$ and $\mathrm{t}=150 \mathrm{~s}$. 
one at $\mathrm{t}=10 \mathrm{~s}$. Far upstream of the cylinder, the sediment flux increases slightly with the increasing bed slope angle. Around the main slope variation $\left(R_{u} / D \approx 0.7\right)$, the flux drastically increases and remains at a high level $\left(q^{*} \approx 4.5\right)$ where the bed angle exceeds the angle of repose. Excluding the area just downstream of the cylinder, the maximum of the sediment flux is located in the two legs around the cylinder. However, compared with $\mathrm{t}=10 \mathrm{~s}$, the HSV legs are wider and the maximum sediment flux is slightly decreasing in time: $q_{\max }^{*}=7.1$ at $\mathrm{t}=60 \mathrm{~s}$ and $q_{\max }^{*}=6.6$ at $\mathrm{t}=150 \mathrm{~s}$. The transverse flux driven by the HSV legs around the cylinder seems thus to decrease in time. This result is coherent with the fact that the erosion rate is higher at the beginning of the scour process (see figure 4). As for $\mathrm{t}=10 \mathrm{~s}$, important differences are observed between the two-phase flow sediment flux and the empirical formula (subfigures 5.h and 5.i).

A better understanding of the sediment transport in the cylinder vicinity can be obtained by investigating the vertical structure of the flow. Figure 6 shows the vertical profiles of the mixture velocity $(\mathrm{U})$, the sediments concentration $(\phi)$ and the dimensionless sediments flux $\left(\pi^{*}=\right.$ $\left.\left(\phi \sqrt{u^{s 2}+v^{s 2}+w^{s 2}}\right) / \sqrt{(s-1) g d^{3}}\right)$ for $\mathrm{t}=10 \mathrm{~s}$ (in blue), $\mathrm{t}=60$ s (in magenta) and $\mathrm{t}=150 \mathrm{~s}$ (in red). The location of each profile is given in subfigure 5.c: at the upstream slope of the scour hole (white circle) and in the HSV legs (squared symbol).

The horizontal dotted lines represent the vertical elevation where the sediments concentration is $\phi=0.08$, allowing to distinguish between bedload and suspended load.

The vertical profile upstream of the pile is located where the bed slope angle is approximatively of $25^{\circ}$ at $\mathrm{t}=10 \mathrm{~s}$ and above the critical angle $\left(\beta>32^{\circ}\right)$ for $\mathrm{t}=60 \mathrm{~s}$ and $\mathrm{t}=150 \mathrm{~s}$. The profiles have a similar shape. The velocity is positive (i.e downstream oriented) just above the bed. This results in a strong downstream oriented bedload sediment flux. Above this dense transport layer, the mixture velocity becomes negative over more than $5 \mathrm{~mm}$ in height for $\mathrm{t}=10 \mathrm{~s}$ and $1 \mathrm{~cm}$ for $\mathrm{t}=60$ and $\mathrm{t}=150 \mathrm{~s}$. The concentration in that region is lower than 0.08 meaning that sediments are transported upstream as suspension. Further away from the bed, the mixture velocity is positive again but the sediments concentration is so low that the associated sediment flux is negligible.

In the HSV legs (bottom panels of Figure 6), the mixture velocity is positive over the entire water depth and the concentration profile is smoother at the bed interface. As a result, there is a positive sediments flux at that location. The peak of sediment flux is located below the vertical position of concentration $\phi=0.08$, meaning that bedload dominates. Nonetheless, an important positive contribution from suspended load is also observed at all times.

In order to better explain the observations obtained from the vertical profiles, Figure 7 shows a vertical plan view in the plane of symmetry upstream of the pile. The velocity vectors and the dimensionless sediments flux $\pi^{*}$ are presented at the same instants: 10s, $60 \mathrm{~s}$ and $150 \mathrm{~s}$. The sediment iso-concentration contours $\phi=0.57$ and $\phi=0.08$ are also plotted in all subfigures to represent the bedload layer. The angle of repose $\left(\beta_{r}=32^{\circ}\right)$ is materialized as the red dashed line. The vertical magenta dashed line represents the position $x / D=-0.7$ of the upstream vertical profile shown in Figure 6.

By examining the bed evolution and the velocity field it can be concluded that the downward flow in front of the pile acts as a vertical jet impinging the sediment bed. This downflow will ultimately be involved in the generation an HSV and both features will lead to sediment erosion. As the scour hole deepens in time the bed slope upstream the pile increases and reaches values higher than the angle of repose $\left(\beta=32^{\circ}\right)$. A competition between the local bed shear stress resulting from the fluid flow above the sediments bed and the downslope gravitational acceleration 

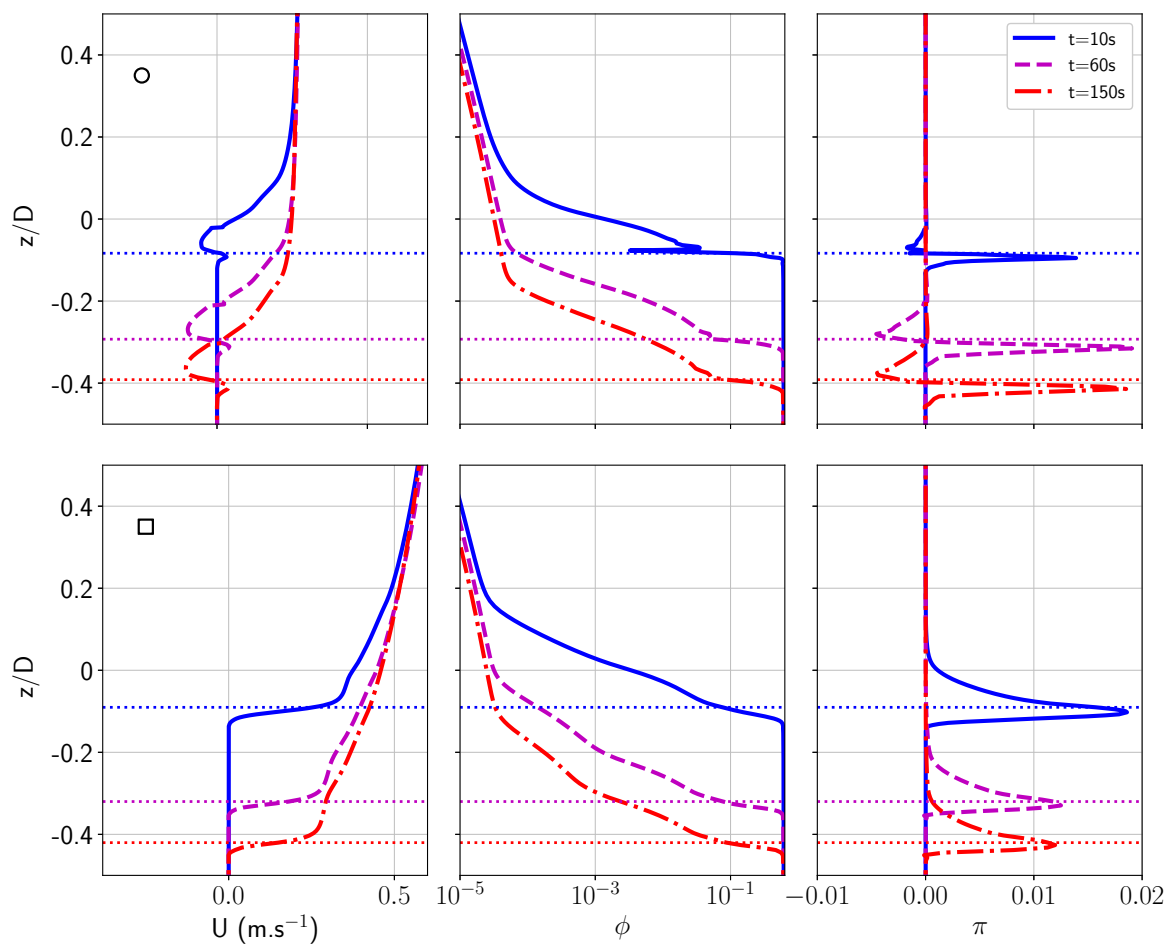

Fig. 6: Vertical profiles of mixture velocity (U), sediments concentration $(\phi)$ and dimensionless sediments flux $(\pi)$ at different locations upstream and around the cylinder and at $\mathrm{t}=10 \mathrm{~s}$ (blue), $\mathrm{t}=60 \mathrm{~s}$ (magenta) and $\mathrm{t}=150 \mathrm{~s}$ (red). The location of each profile is shown in subfigure 5. The horizontal dotted lines represent the vertical elevation where the sediments concentration is $\phi=0.08$.

is taking place in the scour hole. At the upstream part of the scour hole, the slope is mild and the velocity vectors are aligned with the sediments flux (Figure 7 up to $x / D=-1$ ). Closer to the cylinder, in the suspended-load layer, sediments are transported upstream by the HSV while in the bedload layer, sediments are transported downstream by gravity. This result could be corroborated to experimental observation from [Link, 2018] who measured intermittent avalanches in the scour hole. Link et al. [2008, 2012] further observed several slope breaks in their experiments that are probably related to the existence of multiple vortices in the HSV as mentioned by Dargahi [1990]. In the present simulation, the velocity vectors allows to identify a single vortex in the HSV. Its position does not evolve in time and corresponds to the point at which the slope becomes steeper than the angle of repose. In the region between the HSV and the pile, the slope is steeper than the angle of repose meanwhile the velocity vectors and therefore the fluid bed shear stress are in the opposite direction. The downslope gravity flow and the bed shear stress are counteracting each other, but as the angle of repose is exceeded the downslope gravity flow dominates and the net sediment flux is positive (downslope).

The observations presented in the plane of symmetry upstream of the pile are in good agree- 


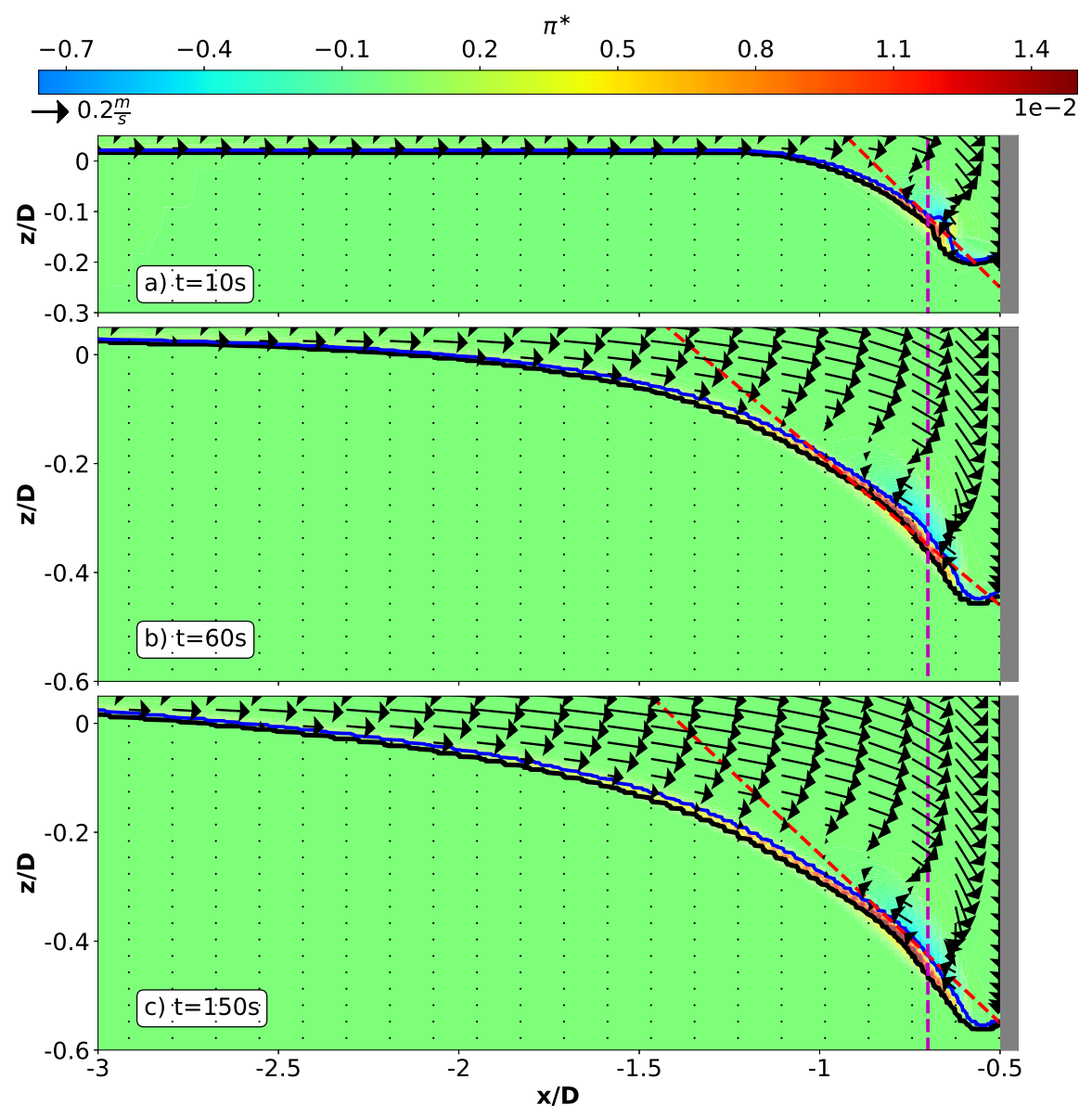

Fig. 7: Flow velocity vectors and sediments flux in the plane of symmetry $(y=0)$ at $t=10 \mathrm{~s}(\mathrm{a}), \mathrm{t}=60 \mathrm{~s}(\mathrm{~b})$ and $t=150 \mathrm{~s}$ (c). The red dashed line represents the avalanche angle of $32^{\circ}$. The black and blue lines represent the sediments isoconcentration $\phi=0.57$ and $\phi=0.08$. The vertical magenta dashed line represents the position $x / D=-0.7$ of the upstream vertical profile shown in Figure 6.

ment with the two-dimensional depth-integrated results showed previously. The importance of both, the slope and the avalanche phenomenon on the sediments transport is confirmed.

\subsection{Downstream of the pile}

Downstream of the cylinder and for $t \geq 60 \mathrm{~s}$ the sediment flux predicted by the two-phase flow model are very significant (see Figure 5.g and Figure 5.k). The strongest fluxes are located up to 1.5 diameter downstream of the pile. Figure 8 shows the same quantities as Figure 6 but at two locations downstream of the pile. The first profile is located in the recirculation cell $(x / D \approx 0.75$, $y / D=0$, star symbol), just downstream of the pile. The mixture velocity shows negative values of 

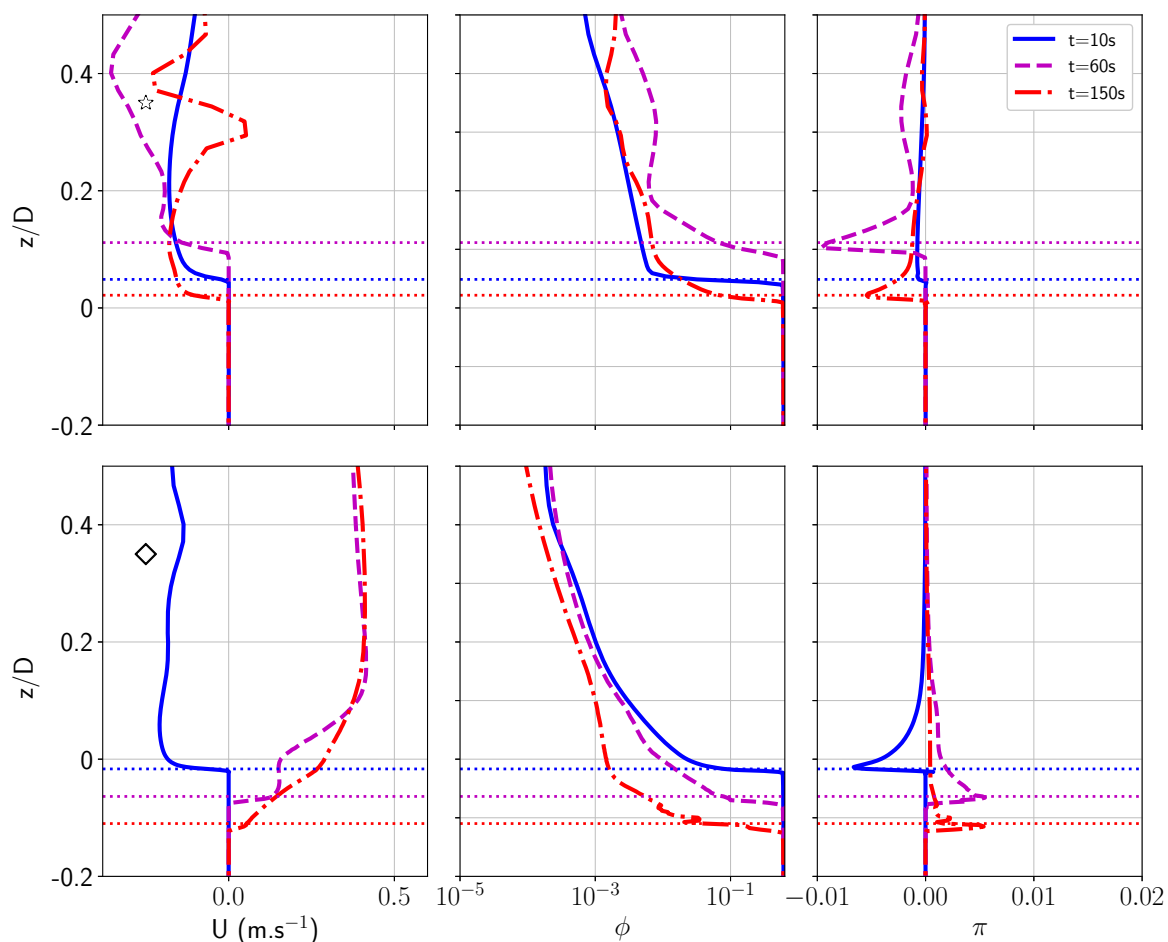

Fig. 8: Vertical profiles of mixture velocity $(U)$, sediments concentration $(\phi)$ and dimensionless sediments flux $(\pi)$ at different locations downstream of the cylinder and at $t=10 \mathrm{~s}$ (blue), $\mathrm{t}=60 \mathrm{~s}$ (magenta) and $\mathrm{t}=150 \mathrm{~s}$ (red). The location of each profile is shown in subfigure 5. The horizontal dotted line represent the vertical elevation of the sediments concentration $\phi=0.08$.

up to $U \approx-0.35 \mathrm{~m} . \mathrm{s}^{-1}$ for the entire vertical profile shown and the concentration is non-negligible $\left(\phi>10^{-3}\right)$ in the water column. As a consequence, there is a negative suspended sediment flux at all times. Although this upstream flux is small at early stage $(\mathrm{t}=10 \mathrm{~s})$, it increase significantly at later stages $(\mathrm{t}=60 \mathrm{~s}$ and $150 \mathrm{~s})$ as sediment concentration increases.

Further downstream $(\mathrm{x} / \mathrm{D} \approx 1.75, \mathrm{y} / \mathrm{D}=0$, diamond symbol) the observations are different. For $\mathrm{t}=10 \mathrm{~s}$, the velocity are negative and the flux is much larger than that closer to the cylinder due to higher suspended sediment concentration. It generates a more important suspended sediments flux toward the cylinder. For $t=60$ and $150 \mathrm{~s}$, the velocity are positive and the sediments are transported downstream by the vortices as bedload and suspended-load. The present results illustrate the complexity of the instantaneous sediment transport downstream of the pile. The sediment concentration profiles at the back of the cylinder drops rapidly away from the bed and suspended load generally dominates bedload, this was already pointed out by Baykal et al. [2015]. 


\section{Discussion}

In Section 5, the role of the local bed slope on sediment transport in the scour hole has been clearly identified. In classical models, the downslope contribution is accounted for as a modification of the critical Shields number in the bed-load flux formula (see Eqn. (25) of Roulund et al. [2005], for instance). This correction is only valid at very low bed slopes and it does not represent the avalanching process. When the bed slope exceeds the angle of repose, an iterative algorithm based on the sediment mass conservation is used to instantaneously limit the bed slope at the angle of repose. This model neglects the avalanche dynamics and the associated relaxation time-scale. In the present two-phase flow model, the avalanche dynamics is implicitly accounted for by using the dense granular flow rheology for the sediment phase. Interestingly, the numerical solution shows that the bed slope can locally exceed the angle of repose in the scour hole very near the pile. This is due to the competition between the fluid bed shear stress associated with the up-slope flow generated by the HSV and the downslope sediment flow. This can not be predicted by classical single-phase flow sediment transport models and illustrates the need for a new modeling approach for this phenomenon in engineering models.

It is possible to use the present two-phase flow model results to infer the local relationship between the sediment fluxes, the local fluid bed shear stress and the local bed slope in the scour configuration. Figure 9.a shows the computed depth-integrated dimensionless sediment flux $q^{*}$ as a function of the Shields parameter along the plane of symmetry $\left(\Gamma=0^{\circ}\right)$. The local sediment flux and Shields parameter are averaged over $10 \mathrm{~s}$ of dynamics around time $t=60 \mathrm{~s}$. The results are compared with the fit given by Eqn. (42) obtained with the same two-phase flow model under unidirectional and uniform flow conditions (red line, see Section 3). The two red dashed line represents $\pm 100 \%$ error with respect to the best-fit. Such confidence interval is considered as reasonable for steady and uniform flow conditions [Gomez and Church, 1989].

The dimensionless sediment flux values are colored by their spatial distance from the inlet. The empty black symbols represent reference points to facilitate the interpretation. At the inlet (dark blue dots, cross symbol), where the flow is not influenced by the presence of the pile, the value of the local Shields number and the dimensionless sediment flux are very close to the uniform flow case $(\theta=0.19)$. Moving downstream $(\mathrm{x} / \mathrm{D}=-2.5$, upward-triangle symbol), both the dimensionless sediment flux and the Shields parameter are decreasing due to the adverse pressure gradient generated by the presence of the cylinder. Despite the fact that the values present a small scatter, they remain within a factor two confidence intervals with respect to the uniform and steady flow best-fit. When getting closer to the cylinder $(\mathrm{x} / \mathrm{D}=-1.5$, downward-triangle symbol), the sediment flux and the Shields number slightly increase and are very close to the unidirectional steady flow case. This can probably be explained by the increase of the local bed slope (see Figure 7) and will be discussed later. Very close to the cylinder ( $\mathrm{x} / \mathrm{D} \in[-1.5 ;-0.7]$, circle symbol), the sediment flux increases drastically by one order of magnitude while the Shields number drops to almost zero. This is the region where the avalanching occurs, the dimensionless sediment flux strongly deviates from the uniform and steady solution: the sediment flux is not related to the fluid bed shear stress, most probably because of the strong downslope gravitational effect and of the avalanching.

Downstream of the pile $(x / D \in[0.5 ; 1.75]$ (star and losange symbols), the dimensionless sediment flux slowly decreases from 10 to 5 while the Shields number oscillates between 0.15 to 0.5 . In this region, the lee-wake vortices dominate the hydrodynamics and the sediment flux is not related to the local fluid bed shear stress. Instead, the sediment flux is dominated by the 
suspended load (see Figure 8) either due to local pick-up of sediments from the bed or to sediments advected from upstream. This explains the poor correlation between the sediment flux and the local Shields number as well as the deviation from the best-fit. Further downstream ( $x / D \geq 3$, from orange to red dots, hexagonal symbol), the dimensionless sediment flux and the Shields parameter decrease due to the weakening of the lee-wake vortices. The sediment flux become closer to the best-fit (factor 2 error only) and the sediment flux correlates again with the local Shields number. The overestimation of the sediment flux can be explained by the enhanced suspended-load observed in Figure 8.

To summarize, at the downstream side of the pile, the dimensionless sediment flux deviation from the uniform and steady state case can be explained by the influence of the lee-wake vortices and the enhanced suspended-load. Upstream of the pile, the deviation from the uniform and steady state case is probably due to the downslope gravitational effect and the avalanching process.

In order to infer the dependency of the local sediment flux to the bed slope, the dimensionless sediment flux along the plane of symmetry is plotted in Figure 9.b as a function of the local bed slope angle $\beta$ at $\mathrm{t}=60 \mathrm{~s}$. The results are only presented between $x / D=-2$ and the upstream edge of the pile and are colored with the same colobar as the one used in Figure 9.a. The two-phase flow model results exhibit a linear dependency on the bed slope for $\beta \leq 23^{\circ}$. As highlighted by the magenta line, a simple linear function of the bed slope angle reproduces fairly well the numerical results: $q^{*} \propto \beta$ for $\beta \in\left[5^{\circ}-25^{\circ}\right]$. The good collapse of the sediment flux with the bed slope and the poor correlation of the sediment flux with the bed shear stress (9.a) demonstrate that the sediment flux in the region just upstream of the pile is dominated by the gravity rather than by the bed shear stress. For $\beta \geq 23^{\circ}$, the sediment flux predicted by the two-phase flow model non-linearly increases with the bed slope angle before reaching a maximum value at $\beta \approx 35^{\circ}$. The local bed slope is on the order or higher than the static friction angle of the $\mu(I)$ rheology meaning that avalanches is taking place. This numerical result is supported by experimental observation from Link [2018] and further confirms that the $\mu(I)$ rheology is probably the best choice for the granular stress model as it is more accurate to predict avalanches. Above this angle the sediment flux decreases drastically with the bed slope angle up to $\beta \approx 45^{\circ}$. In this region, avalanches occurs but sediments are also transported perpendicular to the symmetry plane by the HSV. Very close to the pile, both, the sediment flux and the bed slope angle tend toward zero.

The results presented above show that a competition between fluid bed shear stress driven and gravity driven sediment transport occurs at bed angles lower than the angle of repose $\left(\beta \approx 23^{\circ}\right)$. The relationship between the sediment flux and the bed slope is linear below this value. Above this angle the sediment flux increases nonlinearly with the bed slope while the Shields number vanishes showing that the sediment flux only result from the action of gravity. This changes the vision of the problem and opens new perspectives on modeling the avalanching effect in sediment transport models.

\section{Summary and conclusion}

In this paper, the first three-dimensional Eulerian-Eulerian two-phase flow simulation of scour around a cylinder has been presented. The model has been firstly validated against existing experimental data for the hydrodynamics and the morphodynamics. The relationship between the dimensionless sediment transport flux and the Shields number for uniform and steady-flow 

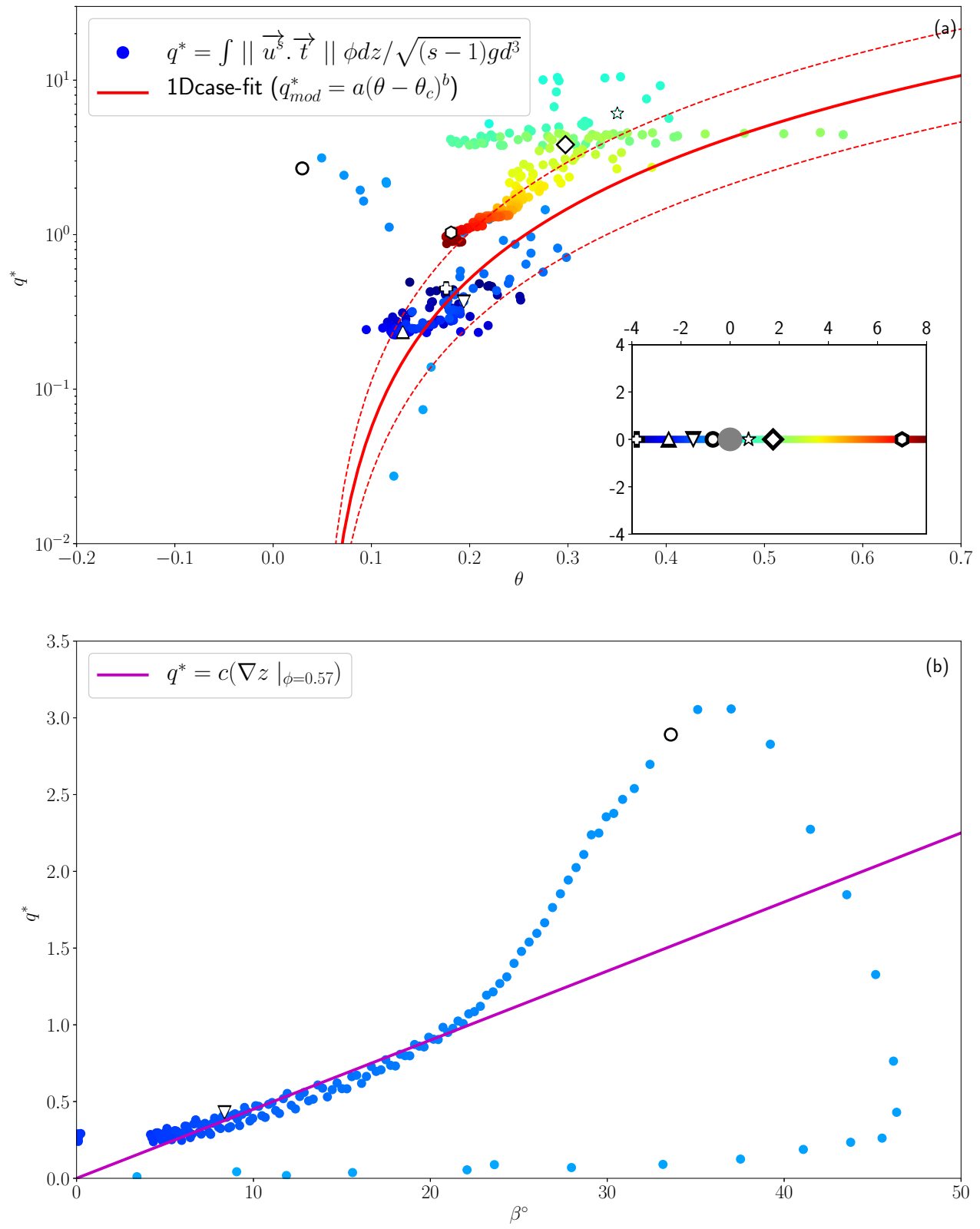

Fig. 9: (a): Dimensionless depth-integrated sediment flux $q^{*}$ (colored by its spatial distance from the inlet) as a function of the Shields parameter along the plane of symmetry $\left(\Gamma=0^{\circ}\right)$. The results are averaged over $10 \mathrm{~s}$ of dynamics around time $\mathrm{t}=60 \mathrm{~s}$. The red line is the uniform flow conditions fit given by Eqn. (42) and the $\phi_{0}=0.08$ shear stress definition. The two red-dashed line represents the $\pm 100 \%$ error with respect to the uniform-fit.

(b): Dimensionless sediment flux $q^{*}$ (colored using the colorbar from (a), but restricted to $-2<x / D<-0.5$ ) as a function of the local slope angle $\beta$ along the plane of symmetry $\left(\Gamma=0^{\circ}\right)$ at $\mathrm{t}=60 \mathrm{~s}$. The magenta line is a linear function of the bed slope angle and is able to represent $q^{*}=f(\beta)$ for $\beta \in\left[5^{\circ}-25^{\circ}\right]$. 
configuration using the two-phase flow approach has been established to serve as a reference to analyze the $3 \mathrm{D}$ effects in the scour simulation. The best-fit of a power-law of the excess shields number results shows very good agreement with the literature for Shields numbers as low as 0.1. These results further demonstrate the capabilities of the two-phase flow approach to deal with sediment transport over a wide range of sediment transport regimes from bed-load to sheetflows. A new methodology to determine the bed shear stress in complex flow configurations is proposed. The mixture shear stress computed at the elevation of the iso-concentration $\phi_{0}=0.08$ (corresponding to top of the bedload layer) is used to define the local Shields number in nonuniform unsteady flow configurations.

Concerning the three-dimensional scour simulation, the good agreement obtained between the numerical results and the available measurements demonstrates the applicability of two-phase flow models to complex sediment transport problems such as scour. The temporal evolution of both, bed morphology and erosion depth, is almost quantitatively reproduced. This allows us to further analyze the numerical results in terms of local correlation between sediment flux, local bed shear stress and local bed slope. Upstream of the pile, the dimensionless flux deviation from the transport law under uniform and steady flows is within a factor two, except in the scour hole, where it deviates by almost one order of magnitude. In this region, the sediment flux results from a competition between the fluid bed shear stress and the downslope gravity effect. Close to the cylinder, the sediment flux is fully correlated with the local bed slope demonstrating that avalanching is dominating. According to our numerical results, in the major part the scour hole, the sediment flux is not correlated with the bed shear stress only. The empirical laws, on which the classical sediment transport models are build, are therefore not accurate. The numerical simulation shows that the downslope gravitational effect becomes very significant at a rather low bed slope angle with a linear dependency of the sediment flux to the bed-slope angle below $\beta \approx 23^{\circ}$ and a nonlinear dependency above this value. These results change the vision of the problem and provides new perspectives on modeling the effect of the bed slope and the avalanching process. Further work is needed to propose new parameterizations that reproduce more accurately the in fluence of the bed-slope.

Concerning the downstream side of the pile, the two-phase flow results suggests that suspendedload is dominant in both the HSV legs and the lee-wake vortices. This result is consistent with previous work on this topic using single-phase flow model [Stahlmann et al., 2013; Baykal et al., 2015].

The main limitation of the two-phase flow model for scour modeling stand probably within the URANS approach. The present k- $\omega 2006$ turbulence model could be improved following Mathieu et al. [2019] recommendations in order to more accurately predict the erosion and the morphodynamics where the interactions between the fluid vortices and the sediment bed are important. Improving the particle presence feedback on the turbulence in the near bed region seems to be the key to improve results. However, this is a very fundamental problem in fluid mechanics and in multiphase flows that even in the simplest configurations (i.e homogeneous isotropic turbulence) is not fully understood. The present work illustrates the capability and limits of the state-of-the-art parametrization for Eulerian-Eulerian model. This point clearly deserves future investigation but we strongly believe that the $3 \mathrm{D}$ scour configuration is not the most appropriate, partly due to the complexity of the flow hydrodynamics and partly due to the lack of experimental data. Moreover, the URANS approach is unable to accurately reproduce the 
actual dynamics of the HSV such as the bimodal oscillation or the existence of multiple vortices. In the lee-wake, the URANS approach is certainly not perfect to predict accurately the vortex shedding and the interaction between these vortices and the sediment dynamics. The natural extension of the present work would be to perform two-phase flow Large Eddy Simulation (LES) of this problem. This approach has been recently applied to unidirectional sheet flows by Cheng et al. [2018] and the application to 3D scour will be carried out in future work.

\section{Acknowledgment}

We would like to thank Oscar Link for the helpful discussions. This work was supported by the french national programme EC2CO-LEFE MODSED. The authors would also like to acknowledge the financial support from the Région Rhône-Alpes through the CMIRA COOPERA project and the ExploraPro grant of J. Chauchat. This research was partially funded by the ANR SEGSED project (ANR-16-CE01-0005-03). Most of the computations presented in this paper were performed using the Froggy platform of the CIMENT infrastructure (https://ciment.ujfgrenoble.fr), which is supported by the Rhône-Alpes region (GRANT CPER07-13 CIRA) and the Equip@Meso project (reference ANR-10-EQPX-29-01) of the programme Investissements d'Avenir supervised by the Agence Nationale pour la Recherche and the GENCI infrastructure under the allocations DARI x2015012153, x2016017567, A0020107567, A0040107567 and A0060107567. Z. Cheng and T-J. Hsu are supported by NSF of USA (OCE-1635151).

We are grateful to the developers involved in OpenFOAM who are the foundation of the model presented in this paper.

\section{Appendix A: Hydrodynamic validation of the flow around a vertical cylinder mounted on a flat bed}

\section{Hydrodynamic setup, computational mesh, boundary conditions}

The numerical domain used for the hydrodynamic validation is a three-dimensional box with a stream-wise length $L x=12 \mathrm{D}$, a span-wise length $\mathrm{Ly}=8 \mathrm{D}$ and a height $\mathrm{H}=\mathrm{D}$ where $\mathrm{D}=53.6 \mathrm{~cm}$ is the pile diameter. This is the same configuration than the Rigid-Bed (RB) case presented in Roulund et al. [2005]. The Reynolds number based on the pile diameter is $R e_{D}=\bar{U} D / v^{f}=1.7 \times$ $10^{5}$, where the mean flow velocity is $\bar{U}=0.326 \mathrm{~m} \cdot \mathrm{s}^{-1}$.

The computational domain is discretized using a unstructured mesh, refined around the cylinder and at the bottom boundary. The mesh refinement area around the cylinder is axisymmetric. The mesh characteristics are given in Table 6 . The boundary conditions are identical to the ones used

Table 6: Mesh characteristics for the RB configuration

\begin{tabular}{cc}
\hline Total number of cells & 2451264 \\
Number of cells around the cylinder perimeter & 512 \\
Horizontal resolution around the cylinder perimeter $(\mathrm{m})$ & $3.25 \times 10^{-3}$ \\
Number of cells across the water depth & 32 \\
Vertical cell aspect ratio & 1.196 \\
Bottom cell height $(\mathrm{m})$ & $4.15 \times 10^{-4}$ \\
\hline
\end{tabular}


by Roulund et al. [2005]:

(i) At the inlet, profiles obtained from a unidirectional vertical simulation driven by a pressure gradient are imposed for $\boldsymbol{u}^{f}, k$ and $\omega$, whereas zero transverse velocities are prescribed.

(ii) At the outlet, zero-gradient conditions (Neumann conditions, $\partial / \partial_{n}=0$ ) are specified for all quantities, except for the reduced pressure for which a homogeneous Dirichlet boundary condition is imposed $\left(p^{*}=0\right)$. For the velocities, a homogeneous Neumann boundary condition is used when the velocity vector points outside of the domain at the outlet, and a homogeneous Dirichlet boundary condition $\left(\boldsymbol{u}^{f}=0\right)$ is used otherwise.

(iii) At the top surface of the computational domain, Neumann conditions are applied for $\mathrm{k}$ and $\omega$ and for the three components of the velocity. As in Roulund et al. [2005] work, the model does not have a free surface.

(iv) On the sides, cyclic conditions are used.

(v) At the walls (bottom and cylinder), zero velocity (no-slip) is imposed for the three components. Consistently with the small non-dimensional distance to the wall $\left(z^{+}=z u_{*} / v^{f} \approx 5\right)$, a very small turbulent kinetic energy is specified for $k\left(\mathrm{O}\left(10^{-6} \mathrm{~m}^{2} \cdot \mathrm{s}^{-2}\right)\right)$. The conditions for $\omega$ are specified using a wall function. In order to account for the flume bottom roughness, a roughness height of $\mathrm{k}_{s}=2.68 \times 10^{-3} \mathrm{~m}$ is imposed at the bottom wall thanks to the tuned $\omega$-wall function described in Roulund et al. [2005]. For the cylinder, the classical wall function from openFOAM is used (omegaWallFunction).

For the initial condition, the unidirectional vertical solution used for the inlet is imposed over the entire numerical domain.

Results
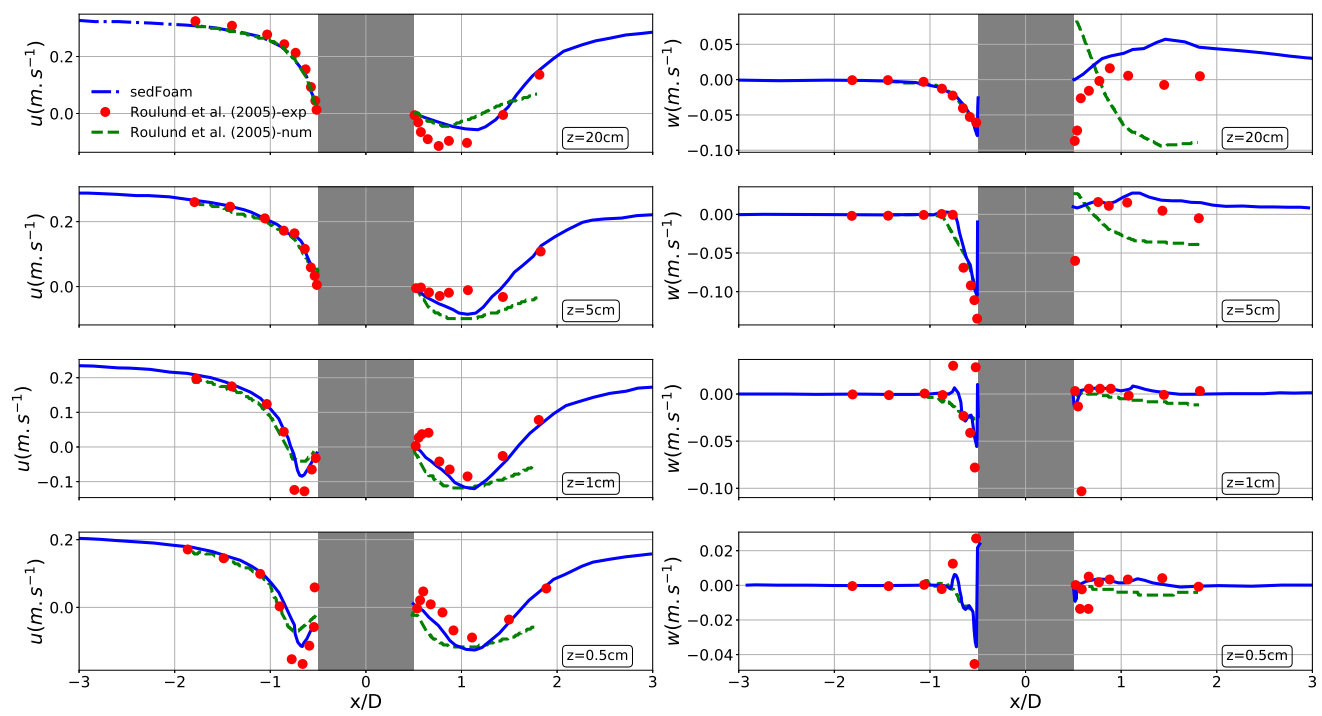

Fig. 10: Horizontal and vertical velocities, u (left panel) and w (right panel), in the plane of symmetry at different distances from the bed. 
Figure 10 shows a comparison of the RB hydrodynamic results with Roulund et al. [2005]'s experimental and numerical results for longitudinal profiles of stream-wise (Figure 10, left panel) and wall-normal (Figure 10, right panel) velocities in the plane of symmetry at different elevations from the bed $(0.5 \mathrm{~cm}, 1 \mathrm{~cm}, 5 \mathrm{~cm}$ and $20 \mathrm{~cm})$. The results have been averaged over 10 vortex shedding periods corresponding to approximatively 60 seconds of dynamics. The flow upstream of the pile is in good agreement with Roulund et al. [2005] experimental data. The HSV, defined as the area in front of the cylinder where the longitudinal velocities are negative, is very well captured (see Figure 10, left panel, at 0.5 and $1 \mathrm{~cm}$ height). Downstream the pile, the change of sign in the velocities shows that there is a counterclockwise recirculation cell. This cell has the correct size compared with experimental data. The counterclockwise recirculation cell was not reproduced by Roulund et al. [2005] steady numerical simulations (see Figure 10, right panel) which confirms the importance of unsteady flow simulations for this configuration (Stahlmann et al. [2013]; Baykal et al. [2015]).

It must be pointed out that a shift is observed for the wall-normal velocities downstream of the pile at $20 \mathrm{~cm}$ in the water layer, compared with Roulund et al. [2005] experimental results. This is probably due to rigid-lid effects and accounting for the free surface could improve the accuracy of the results, as shown in Zhou [2017].

\section{Horseshoe vortex}

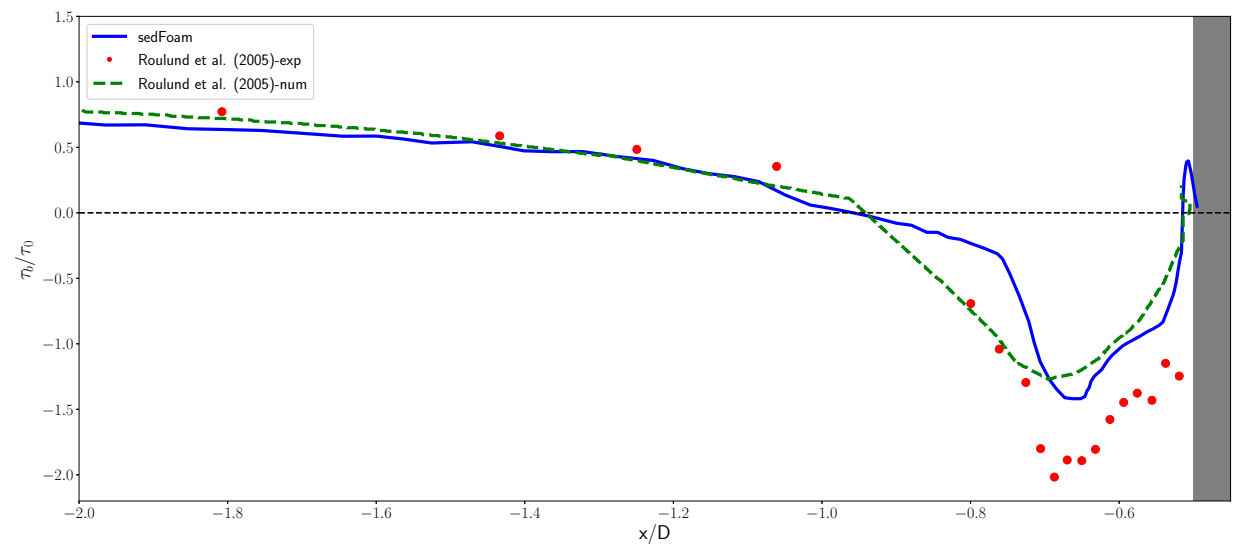

Fig. 11: Comparison between the bed shear stress amplification $\tau_{b} / \tau_{0}$ along the longitudinal axis predicted by SedFoam (blue dots) and Roulund et al. [2005] experimental and numerical results.

Figure 11 shows a comparison between the bed shear stress amplification $\tau_{b} / \tau_{0}$ along the longitudinal axis in the plane of symmetry predicted by the model (blue line) and Roulund et al. [2005] experimental and numerical results. The bed shear stress amplification is computed as the ratio between the local bed shear stress and its value at the inlet, where the flow is undisturbed by the cylinder presence. The local bed shear stress $\tau_{b}$ is computed as:

$$
\tau_{b}=\sqrt{\tau_{x z}^{f^{2}}+\tau_{y z}^{f^{2}}} \times \operatorname{sign}\left(\tau_{x z}^{f}\right)
$$

where $\tau_{x z}^{f}$ and $\tau_{y z}^{f}$ are the components of the fluid shear stress tensor $\tau^{f}$ at the bottom. In Figure 
11 , the zero-crossing of the bed shear stress amplification in front of the pile, between $x / D=-1$ and $x / D=-0.5$, shows the location of the HSV. The two-phase flow model results are in very good agreement with Roulund et al. [2005] results outside of the HSV region $(x / D<-1)$. However, inside the HSV region the negative bed shear stress amplification is underestimated. The difference with amplification found in Roulund et al. [2005] experimental work is about 25\%. This is slightly improved compared with the findings of Roulund et al. [2005] and Baykal et al. [2015]'s numerical work, were the difference was more than 30\%. As mentioned in Roulund et al. [2005] and Baykal et al. [2015], no clear explanation can be provided for these discrepancies between experimental observations and numerical predictions.

\section{Lee-wake vortices}

The regime of the lee-wake vortices generated downstream of a vertical cylinder in a unidirectional flow depends on the pile Reynolds number of the flow. Here, $\operatorname{Re}_{D}=1.7 \times 10^{5}$, the flow is in the so-called subcritical regime. The wake flow is expected to be completely turbulent with vortices shed alternatively at each side of the pile [Sumer et al., 2006].
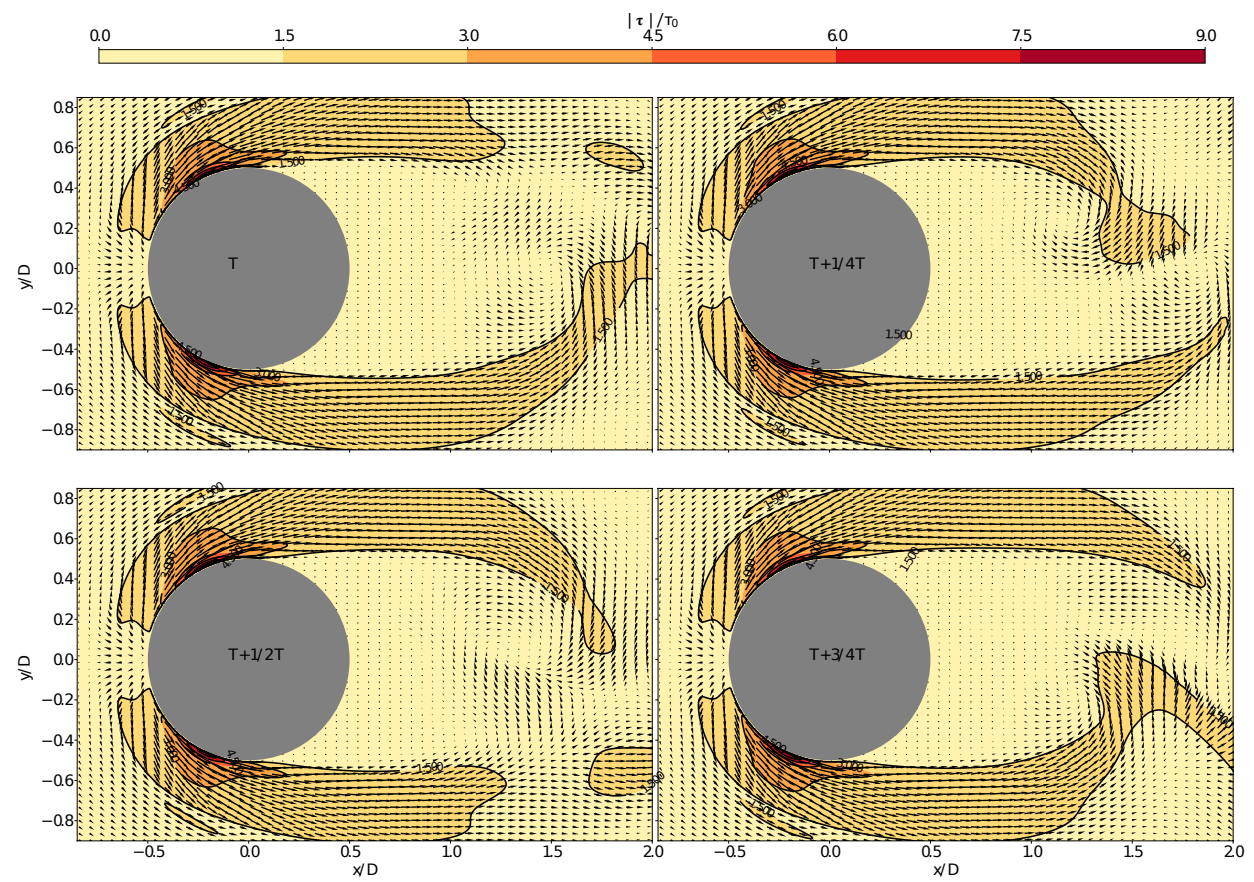

Fig. 12: Bed shear stress vectors and contour lines of the magnitude of bed shear stress amplification over one period of vortex shedding at the bottom. $\mathrm{T} \approx 6 \mathrm{~s}$ is the vortex-shedding period.

Figure 12 shows the bed shear stress vectors and the contour lines of the magnitude of the bed shear stress amplification over one period of vortex shedding at the bottom of the RB case.

The magnitude of the bed shear stress is calculated as $|\tau|=\sqrt{\tau_{x z}^{f^{2}}+\tau_{y z}^{f^{2}}}$.

The position of the maximum bed shear stress in the cylinder vicinity can be found around $\Gamma=65^{\circ}$ 
where $\Gamma$ is the angle measured with respect to the upstream $x$-axis. In Roulund et al. [2005] and Baykal et al. [2015], the magnitude of the bed shear stress amplification is compared with the experiments of Hjorth [1975], where the pile Reynolds number is $R e_{D}=1.5 \times 10^{4}$, i.e. one order of magnitude lower than the present configuration. Despite the diffence in term of Reynolds number, it is shown in Figure 17 of Roulund et al. [2005] and in Figure 3 of Baykal et al. [2015] that their numerical simulations also predict the maximum bed shear stress around $\Gamma=65^{\circ}$. The discrepancies between the numerical prediction and the experimental observation of Hjorth [1975] (where $\Gamma=45^{\circ}$ ) remain unexplained by Roulund et al. [2005] and Baykal et al. [2015].

Again, because of the diffence in term of Reynolds number between the configurations, no definitive comparison can be provided in term of bed shear stress amplification but the order of magnitude found with SedFoam is similar to what is reported in Hjorth [1975] configuration $\left(\max \left(|\tau| / \tau_{0}\right) \approx 9\right)$.

Figure 12 also shows the unsteady behavior of the flow downstream of the cylinder. In the four panels, snapshots during a vortex-shedding period (T) are shown. The generation of two vortices at the opposite sides of the pile, one at $t=0[T]$ (Figure 12 top left panel) and the other at $\mathrm{t}=\mathrm{T}+\mathrm{T} / 2$ is clearly visible. Here, the vortex-shedding period is approximatively $6 \mathrm{~s}$. Although higher than the theoretical Strouhal value $\left(S_{t r}=0.2\right)$, the present Strouhal number $\left(S_{t r}=0.27\right)$ is in good agreement with the one reported in Baykal et al. [2015].

\section{Discussion on Rigid-Bed hydrodynamics}

The detailed study of the hydrodynamics around the cylinder such as the HSV and the leewake vortices demonstrates the good behavior of the proposed model to reproduce the flow around a vertical cylinder in a unidirectional current without sediments. The rigid-lid assumption might be questionable as all experiments have been carried-out in open-channels. Indeed, constraining the domain height could be an over-simplification of the problem. It is usually accepted that when the Froude number is lower than about 0.2 the free surface deformation near the structure is negligible [Roulund et al., 2005]. Even in this limit, the rigid-lid treatment is only an approximation. In the results presented above, the shift of the wall-normal velocities downstream of the pile near the free surface compared with Roulund et al. [2005] experimental data can be attributed to the fact that the free surface deformation is not accounted for. This has been shown by Zhou [2017] who performed rigid-lid and free-surface computations of Roulund et al. [2005] configuration. However, at the upstream side of the pile, in the region where the HSV is generated and the scour occurs, the rigid-lid approximation has almost no influence on the numerical results. It is therefore reasonable to use this approximation to perform 3D scour simulation using the two-phase flow model.

\section{References}

Amoudry, L., Hsu, T.-J., Liu, P.-F., 2008. Two-phase model for sand transport in sheet flow regime. Journal of Geophysical Research: Oceans 113 (C3).

Amoudry, L., Liu, P.-F., 2009. Two-dimensional, two-phase granular sediment transport model with applications to scouring downstream of an apron. Coastal Engineering 56 (7), 693-702.

Andreotti, B., Forterre, Y., Pouliquen, O., 2013. Granular Media: Between Fluid and Solid. Cambridge.

Bagnold, R. A., 1956. The flow of cohesionless grains in fluids. Philosophical Transactions of the Royal Society of London. Series A, Mathematical and Physical Sciences 249 (964), 235-297.

Baykal, C., Sumer, B. M., Fuhrman, D. R., Jacobsen, N. G., Fredsøe, J., 2015. Numerical investigation of flow and scour around a vertical circular cylinder. Philosophical Transactions of the Royal Society of London A: Mathematical, Physical and Engineering Sciences 373 (2033), 20140104. 
Benavides, A., van Wachem, B., 2008. Numerical simulation and validation of dilute turbulent gas-particle flow with inelastic collisions and turbulence modulation. Powder Technology 182 (2), 294-306.

Boyer, F. m. c., Guazzelli, E., Pouliquen, O., Oct 2011. Unifying suspension and granular rheology. Phys. Rev. Lett. 107, 188301.

Breusers, H., Nicollet, G., Shen, H., 1977. Local scour around cylindrical piers. Journal of Hydraulic Research 15 (3), 211-252.

Breusers, H., Raudkivi, A., 1991. Scouring. Balkema Rotterdam.

Chauchat, J., 2018. A comprehensive two-phase flow model for unidirectional sheet-flows. Journal of Hydraulic Research, 1-14.

Chauchat, J., Cheng, Z., Nagel, T., Bonamy, C., Hsu, T.-J., 2017. Sedfoam-2.0: a 3-d two-phase flow numerical model for sediment transport. Geoscientific Model Development 10 (12), 4367.

Chauchat, J., Médale, M., 2014. A three-dimensional numerical model for dense granular flows based on the rheology. Journal of Computational Physics 256 (0), $696-712$. URL http://www.sciencedirect.com/science/article/pii/S0021999113006098

Chen, C., Wood, P., 1985. A turbulence closure model for dilute gas-particle flows. The canadian journal of chemical engineering 63 (3), 349-360.

Cheng, Z., Hsu, T.-J., Calantoni, J., 2017. Sedfoam: A multi-dimensional eulerian two-phase model for sediment transport and its application to momentary bed failure. Coastal Engineering 119, 32-50.

Cheng, Z., Hsu, T.-J., Chauchat, J., 2018. An eulerian two-phase model for steady sheet flow using large-eddy simulation methodology. Advances in Water Resources 111, 205-223.

Coleman, N. L., 1970. Flume studies of the sediment transfer coefficient. Water Resour. Res. 6, 801-809.

Danon, H., Wolfshtein, M., Hetsroni, G., 1977. Numerical calculations of two-phase turbulent round jet. International Journal of Multiphase Flow 3 (3), 223-234.

Dargahi, B., 1990. Controlling mechanism of local scouring. Journal of Hydraulic Engineering 116 (10), $1197-1214$

Deshpande, S. S., Anumolu, L., Trujillo, M. F., 2012. Evaluating the performance of the two-phase flow solver interfoam. Computational science \& discovery 5 (1), 014016

Deutsch, E., Simonin, O., 1991. Large eddy simulation applied to the motion of particles in stationary homogeneous fluid turbulence. Turbulence Modification in Multiphase Flows ASME-FED 110, 35 - 42

Ding, J., Gidaspow, D., 1990. A bubbling fluidization model using kinetic theory of granular flow. AIChE journal 36 (4), 523-538.

Dohmen-Janssen, C. M., Hassan, W. N., Ribberink, J. S., 2001. Mobile-bed effects in oscillatory sheet flow. Journal of Geophysical Research: Oceans 106 (C11), 27103-27115.

Engelund, F., Fredsøe, J., 1976. A sediment transport model for straight alluvial channels. Hydrology Research 7 (5), 293-306

Escauriaza, C., Sotiropoulos, F., 2011. Lagrangian model of bed-load transport in turbulent junction flows. Journal of Fluid Mechanics 666, 36-76.

Exner, F. M., 1920. Zur physik der dünen. Hölder

Exner, F. M., 1925. Uber die wechselwirkung zwischen wasser und geschiebe in flüssen. Akad. Wiss. Wien Math. Naturwiss. Klasse 134 (2a), 165-204.

Forterre, Y., Pouliquen, O., 2008. Flows of dense granular media. Annual Review of Fluid Mechanics 40, 1-24. URL http://link. aip.org/link/?PHF/17/103301/1

GDRmidi, 2004. On dense granular flows. The European Physical Journal E 14, 341-365.

Gomez, B., Church, M., 1989. An assessment of bed load sediment transport formulae for gravel bed rivers. Water Resources Research 25 (6), 1161-1186.

Hjorth, P., 1975. Studies on the nature of local scour. Inst. för Teknisk Vattenresurslära, Lunds Tekniska Högskola, Lunds Univ.

Hsu, T.-J., Jenkins, J. T., Liu, P. L.-F., 2004. On two-phase sediment transport: sheet flow of massive particles. In Proceedings of the Royal Society of London A: Mathematical, Physical and Engineering Sciences. Vol. 460. The Royal Society, pp. 2223-2250.

Jenkins, J. T., Hanes, D. M., 1998. Collisional sheet flows of sediment driven by a turbulent fluid. Journal of Fluid Mechanics 370, 29-52.

Jenkins, J. T., Savage, S. B., 1983. A theory for the rapid flow of identical, smooth, nearly elastic, spherical particles Journal of Fluid Mechanics 130, 187-202.

Jha, S. K., Bombardelli, F. A., 2009. Two-phase modeling of turbulence in dilute sediment-laden, open-channel flows. Environmental fluid mechanics 9 (2), 237.

Johnson, P. C., Jackson, R., 3 1987. Frictional-collisional constitutive relations for granular materials, with application to plane shearing. Journal of Fluid Mechanics 176, 67-93.

Jop, P., Forterre, Y., Pouliquen, O., 2006. A constitutive law for dense granular flows. Nature 441, 727- 730. URL http://dx.doi.org/10.1038/nature04801 
Kranenburg, W. M., Hsu, T.-J., Ribberink, J. S., 2014. Two-phase modeling of sheet-flow beneath waves and its dependence on grain size and streaming. Advances in water resources 72, 57-70.

Lee, C.-H., Low, Y. M., Chiew, Y.-M., 2016. Multi-dimensional rheology-based two-phase model for sediment transport and applications to sheet flow and pipeline scour. Physics of Fluids 28 (5), 053305.

Link, O., 2018. Personal communication.

Link, O., González, C., Maldonado, M., Escauriaza, C., 2012. Coherent structure dynamics and sediment particle motion around a cylindrical pier in developing scour holes. Acta Geophysica 60 (6), 1689-1719.

Link, O., Pfleger, F., Zanke, U., 2008. Characteristics of developing scour-holes at a sand-embedded cylinder. International Journal of Sediment Research 23 (3), 258-266.

Mathieu, A., Chauchat, J., Bonamy, C., Nagel, T., 2019. Two-phase flow simulation of tunnel and lee-wake erosion of scour below a submarine pipeline. Water $11(8), 1727$.

Maurin, R., Chauchat, J., Frey, P., 2016. Dense granular flow rheology in turbulent bedload transport. Journal of Fluid Mechanics 804, 490-512.

Melville, B. W., Coleman, S. E., 2000. Bridge scour. Water Resources Publication.

Menter, F. R., 1993. Zonal two equation k- omega turbulence models for aerodynamic flows. AIAA paper $2906,1993$.

Meyer-Peter, E., Müller, R., 1948. Formulas for bed-load transport. IAHR.

Nagel, T., 2018. Numerical study of multi-scale flow-sediment-structure interactions using a multiphase approach. Ph.D. thesis, Grenoble Alpes University.

Pope, S., 1988. An explanation of the turbulent round jet/plane jet anomaly. AIAA Journal 16 (3), 279-281.

Revil-Baudard, T., Chauchat, J., 2013. A two-phase model for sheet flow regime based on dense granular flow rheology. Journal of Geophysical Research: Oceans 118 (2), 619-634.

URL http://dx.doi .org/10.1029/2012JC008306

Revil-Baudard, T., Chauchat, J., Hurther, D., Barraud, P.-A., 3 2015. Investigation of sheet-flow processes based on novel acoustic high-resolution velocity and concentration measurements. Journal of Fluid Mechanics 767, 1-30.

Roulund, A., Sumer, B., Fredsøe, J., Michelsen, J., 2005. Numerical and experimental investigation of flow and scour around a circular pile. Journal of Fluid Mechanics 534, 351-401.

Rusche, H., 2003. Computational fluid dynamics of dispersed two-phase flows at high phase fractions. Ph.D. thesis, Imperial College London (University of London).

Schiller, L., Naumann, A., 1933. Uber die Grundlegenden Berechungen bei der Schwerkraftaufbereitung. Ver. Deut. Ing. 77.

Stahlmann, A., et al., 2013. Numerical and experimental modeling of scour at foundation structures for offshore wind turbines. In: The Twenty-third International Offshore and Polar Engineering Conference. International Society of Offshore and Polar Engineers.

Sumer, B. M., Kozakiewicz, A., Fredsøe, J., Deigaard, R., 1996. Velocity and concentration profiles in sheet-flow layer of movable bed. Journal of Hydraulic Engineering 122 (10), 549-558.

Sumer, B. M., et al., 2006. Hydrodynamics around cylindrical strucures. Vol. 26. World scientific.

Van Rijn, L., 1984. Sediment transport, part ii: Suspended load transport. J. Hydraul. Eng. 110, 1613-1641.

Wilcox, D. C., 2006. Turbulence modeling for CFD, 3rd edition. DCW industries La Canada, CA.

Wilcox, D. C., 2008. Formulation of the k-omega turbulence model revisited. AIAA J 46 (11), 2823-2838.

Wilson, K. C., 1966. Bed-load transport at high shear stress. Journal of the hydraulics division 92 (6), 49-59.

Wilson, K. C., 1987. Analysis of bed-load motion at high shear stress. Journal of Hydraulic Engineering 113 (1), $97-103$.

Wong, M., Parker, G., 2006. Reanalysis and correction of bed-load relation of meyer-peter and müller using their own database. Journal of Hydraulic Engineering 132 (11), 1159-1168.

Yu, X., Hsu, T.-J., Hanes, D. M., 2010. Sediment transport under wave groups: Relative importance between nonlinear waveshape and nonlinear boundary layer streaming. Journal of Geophysical Research: Oceans 115 (C2).

Zhao, M., Cheng, L., Zang, Z., 2010. Experimental and numerical investigation of local scour around a submerged vertical circular cylinder in steady currents. Coastal Engineering 57 (8), 709-721.

Zhou, L., 2017. Numerical modelling of scour in steady flows. Ph.D. thesis, Université de Lyon. 